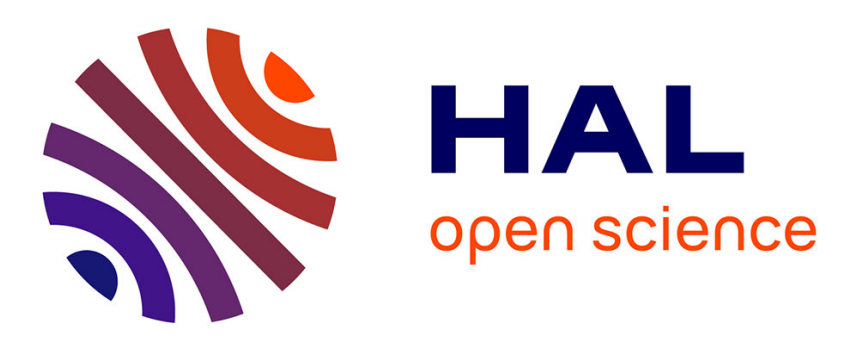

\title{
Thermal buckling response of laminated and sandwich plates using refined 2-D models
}

\author{
R. Vescovini, M. d'Ottavio, L. Dozio, O. Polit
}

\section{To cite this version:}

R. Vescovini, M. d'Ottavio, L. Dozio, O. Polit. Thermal buckling response of laminated and sandwich plates using refined 2-D models. Composite Structures, 2017, 176, pp.313-328. 10.1016/j.compstruct.2017.05.021 . hal-01981940

\section{HAL Id: hal-01981940 \\ https://hal.parisnanterre.fr/hal-01981940}

Submitted on 15 Jan 2019

HAL is a multi-disciplinary open access archive for the deposit and dissemination of scientific research documents, whether they are published or not. The documents may come from teaching and research institutions in France or abroad, or from public or private research centers.
L'archive ouverte pluridisciplinaire HAL, est destinée au dépôt et à la diffusion de documents scientifiques de niveau recherche, publiés ou non, émanant des établissements d'enseignement et de recherche français ou étrangers, des laboratoires publics ou privés. 


\title{
Thermal buckling response of laminated and sandwich plates using refined 2-D models
}

\author{
R. Vescovini, M. D’Ottavio, L. Dozio, O. Polit
}

\section{Introduction}

Thermal loads play an important role in several fields of engineering. Aeronautical and space applications are a well-known example, where the structures commonly experience severe aerodynamic heating and drastic changes of thermal environments. For instance, the launch and the re-entry of space vehicles is typically associated with severe temperature variations. Similarly, thermal loads are developed in supersonic and hypersonic aircrafts as the result of restrained thermal expansion [1].

These aspects, in conjunction with the typical thin-walled architecture of light-weight structures, have made thermal buckling the subject of several investigations in the past. Early works are based on classical lamination theory (CLT) and first order shear deformation theory (FSDT). Reviews of analytical solutions using classical CLT and FSDT are available on several textbooks $[2,3]$. A comprehensive overview of the topic is proposed by Jones [4] with regard to fiber-reinforced composite plates, where CLT is applied and the equivalent mechanical loading concept is

\footnotetext{
* Corresponding author.

E-mail address: michele.d_ottavio@u-paris10.fr (M. D’Ottavio).
}

discussed. Within the context of CLT, Nemeth [5] investigated the thermal buckling response of infinitely long plates, introducing stiffness-weighted thermal-expansion parameters as a useful mean to obtain design charts, whose validity extends to a wide range of laminates. Recently, the effects of non-uniform temperature distribution on thermal buckling have been assessed by $\mathrm{Li}$ et al. [6] using CLT in the context of an energy-based formulation. The work was successively extended to account for random system properties [7]. Still in the context of CLT, variable stiffness plates have been optimized with regard to thermal buckling requirements using finite elements in Ref. [8].

While CLT can be successfully applied to analyze a wide class of thin-plate problems, different strategies are needed to analyze more general plate configurations, where transverse shear deformation effects cannot be neglected. In this sense, a relatively vast amount of research efforts has regarded the development of more refined approaches, FSDT being the simplest one. An early work by Tauchert [9] discusses the derivation of closed-form solutions for anti-symmetric angle-ply plates based on FSDT, while mixed FSDT finite-elements are adopted by Noor and Peters [10] to address the sensitivity of thermal buckling loads to lamination and material properties. Recently, FSDT has been applied also to analyze the thermal buckling of FGM shells [11]. 
High-order shear deformation theories were employed in Refs. $[12,13]$ and closed-form solutions obtained for simply-supported plates subjected to a uniform temperature rise. Erroneous assumptions on the plate constitutive law of Ref. [12] were successively pointed out by Rohwer [14], and results corrected accordingly.

Other refined techniques for the analysis of laminated plates included the high-order mixed formulation and the global/local higher order theory proposed in Refs. $[15,16]$, respectively, while layerwise approaches have been carried out with finite elements in Refs. [17,18].

Despite the vast amount of research papers dealing with composite plates, less studies are available on the thermal buckling response of sandwich plates. A simplified analytical study is proposed in Ref. [19], based on the Ritz method and Libove-Batdorf sandwich theory [20]. A higher-order plate finite element is used in Refs. [21,22] to address the thermal buckling response of sandwich panels, including skew configurations. An analytical approach is developed by Frostig [23] for the thermal buckling and postbuckling based on a high-order approach incorporating the effects of the core flexibility. Equivalent single layer models are discussed by Pradeep et al. [24], and Matsunaga with regard to plates with cross-ply and angle-ply face-sheets [25-27]. These latter studies include a simplified treatment of the pre-buckling deformations on the thermal buckling load, demonstrating their role on the critical temperature prediction.

Three dimensional solutions were developed by Noor and coworkers [28-30] for both laminated and sandwich panels. In these pioneering works, the relevance of pre-buckling deformations was illustrated by providing results for temperature-independent [28] and temperature-dependent [29] properties. Despite their usefulness for deriving benchmark results, three dimensional solution procedures are usually too costly to be used for common design purposes, and more efficient approaches are needed.

In this sense, a powerful strategy for including several theories into one single formulation is given by the unified formulation proposed by Carrera, often denoted with the acronym CUF [31,32]. This kind of variable-kinematic approach has been adopted to analyze the mechanical response of sandwich panels in several works [33-37], while the prediction of the critical temperatures is discussed in Refs. $[38,39]$. Within the same framework, the thermal stability of functionally graded sandwich has been recently assessed [40].

The importance of retaining the transverse normal deformation in the presence of thermal loading and the influence of the actual temperature distribution across the plate thickness was pointed out by Carrera in Refs. [41,42].

In recent works by the authors, the Generalized Unified Formulation proposed by Demasi [43-45] was extended to include a sublaminate approach [36,37] for the bending analysis of sandwich plates with the aim of optimizing the accuracy-to-cost ratio. The resulting approach was indicated as Sublaminate Generalized Unified Formulation (S-GUF).

In the present paper, the S-GUF is developed in the context of a Ritz-based approach [46-48] and its formulation is extended to address the thermal buckling response of laminated plates and sandwich panels.

As opposed to the vast majority of previous studies, the initial stress produced by a uniform thermal loading is considered and the effect of the pre-buckling transverse normal deformation is assessed. This technique, suggested by Noor and Burton [28], introduces the deformed pre-buckling state into the classical eigenvalue problem for determining the critical temperature, which is then solved iteratively.

A more accurate identification of the initial stress state, that accounts for a refined representation of the thermal loading profile, the actual boundary conditions of the plate and, where necessary, temperature-dependent material properties, would require the solution of a more complex non-linear problem and remains out of the scope of this paper.

The results illustrate the excellent accuracy of the predictions in relation with the relatively low number of degrees of freedom. Novel results are finally proposed as a benchmark for future studies.

\section{Formulation}

The formulation is developed for the thermal buckling analysis of composite plates in the framework of the SublaminateGeneralized Unified Formulation (S-GUF). Linear and nonlinear pre-buckling deformations are taken into account. The panels are flat and can be either monolithic or sandwich. A sketch of the panel is provided in Fig. 1, where the reference system together with the typical dimensions of the panel are reported. In particular, $a$ and $b$ denote the length and the width of the panel, respectively, while $h$ is the total thickness. For generality purposes, the sketch refers to a sandwich configuration, where the quantity $h_{\mathrm{f}}$ denotes the thickness of the single face-sheet. In the case of monolithic composite plates, the panel thickness is simply denoted with $h$, while $h_{\mathrm{f}}$ becomes meaningless.

The panel is subjected to a uniform temperature increase, although the formulation could be, in principle, extended to consider nonuniform distributions. The initial heating of the panels which constitutes the pre-buckling condition - happens by assuming fully restrained boundary conditions, where immovable edges are considered. The buckling boundary conditions, as discussed next, can be any sort of combination involving the vanishing or non-vanishing of the three-inplane displacement components.

The formulation is thus characterized by two distinct steps, consisting in the solution of the pre-buckling and buckling condition. When the nonlinear pre-buckling effects are accounted for, the equations are in the form of a nonlinear eigenvalue problem, which is solved iteratively. On the contrary, the classical approach based on a linear pre-buckling analysis leads to the solution of a standard eigenvalue problem.

The Sublaminate-Generalized Unified Formulation is an axiomatic approach for the analysis of composite structures based on the idealization of the structure as an assembly of $N_{k}$ sublaminates. The fundamental ideas of the approach are here reviewed, while a deeper discussion is available in [36,37]. Each sublaminate is a cluster of $N_{p}^{k}$ plies, arbitrarily chosen by the user, where an arbitrary kinematic theory is chosen for describing the displacement field. A fully displacement-based approach is adopted. By collecting the three displacement components of the generic ply $p$ belonging to the sublaminate $k$, the $3 \times 1$ vector $\mathbf{u}^{p, k}$ is defined as:

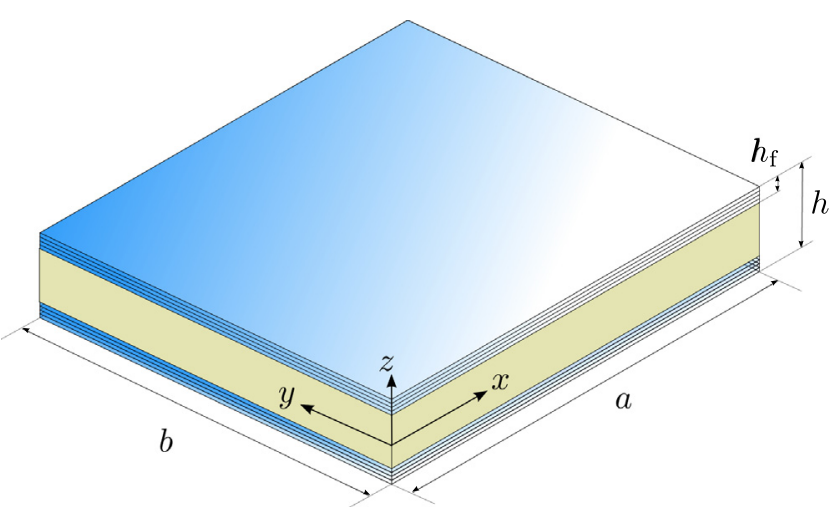

Fig. 1. Plate dimensions and reference system. 
$\mathbf{u}^{p, k}=\left\{\begin{array}{lll}u_{x}^{p, k} & u_{y}^{p, k} & u_{z}^{p, k}\end{array}\right\}^{\mathrm{T}}$

where $u_{r}^{p, k}$ denotes the displacement component along the generic coordinate direction $r=x, y, z$. The Generalized Unified Formulation (GUF) proposed by Demasi [43-45] is adopted as underlying kinematic description for approximating the displacement components of Eq. (1). In particular, following the notation of GUF, the components of Eq. (1) are expressed as:

$$
\begin{cases}u_{x}^{p, k}\left(x, y, z_{p}\right)=F_{\alpha_{u_{x}}}\left(z_{p}\right) u_{x \alpha_{u_{x}}}^{p, k}(x, y) & \alpha_{u_{x}}=0,1, \ldots, N_{u_{x}}^{k} \\ u_{y}^{p, k}\left(x, y, z_{p}\right)=F_{\alpha_{u y}}\left(z_{p}\right) u_{y \alpha_{u y}}^{p, k}(x, y) & \alpha_{u_{y}}=0,1, \ldots, N_{u_{y}}^{k} \\ u_{z}^{p, k}\left(x, y, z_{p}\right)=F_{\alpha_{u_{z}}}\left(z_{p}\right) u_{z \alpha_{u_{z}}}^{p, k}(x, y) & \alpha_{u_{z}}=0,1, \ldots, N_{u_{z}}^{k}\end{cases}
$$

where separation of variables is assumed between the thicknesswise and in-plane directions. In particular, $F_{\alpha_{u_{r}}}$ denotes the thickness function, while $u_{r \alpha_{u_{r}}}^{p, k}$ are the generalized displacement
$\boldsymbol{\varepsilon}_{\Omega}^{p, k}=\mathbf{D}_{\Omega} \mathbf{u}^{p, k} \quad \boldsymbol{\varepsilon}_{\mathrm{n}}^{p, k}=\mathbf{D}_{\mathrm{n}} \mathbf{u}^{p, k}+\mathbf{D}_{z} \mathbf{u}^{p, k}$

where the subscripts $\Omega$ and $\mathrm{n}$ are introduced to denote the in-plane and the normal components, respectively. The two vectors of Eq. (6) read:

$\boldsymbol{\varepsilon}_{\Omega}^{p, k}=\left\{\begin{array}{lll}\varepsilon_{x x}^{p, k} & \varepsilon_{y y}^{p, k} & \gamma_{x y}^{p, k}\end{array}\right\}^{\mathrm{T}} \quad \boldsymbol{\varepsilon}_{\mathrm{n}}^{p, k}=\left\{\begin{array}{lll}\gamma_{y z}^{p, k} & \gamma_{x z}^{p, k} & \varepsilon_{z z}^{p, k}\end{array}\right\}^{\mathrm{T}}$

and the differential matrices of Eq. (6) are defined as:

$\mathbf{D}_{\Omega}=\left[\begin{array}{ccc}\frac{\partial}{\partial x} & 0 & 0 \\ 0 & \frac{\partial}{\partial y} & 0 \\ \frac{\partial}{\partial y} & \frac{\partial}{\partial x} & 0\end{array}\right] \quad \mathbf{D}_{\mathrm{n}}=\left[\begin{array}{ccc}0 & 0 & \frac{\partial}{\partial x} \\ 0 & 0 & \frac{\partial}{\partial y} \\ 0 & 0 & 0\end{array}\right] \quad \mathbf{D}_{z}=\left[\begin{array}{ccc}\frac{\partial}{\partial z} & 0 & 0 \\ 0 & \frac{\partial}{\partial z} & 0 \\ 0 & 0 & \frac{\partial}{\partial z}\end{array}\right]$

In a similar fashion, the nonlinear terms of the strain tensor $\eta_{i j}$ expressed by Eq. (5) are organized as:

$\boldsymbol{\eta}_{\Omega}^{p, k}=\left\{\begin{array}{lll}\frac{1}{2}\left(u_{x, x}^{p, k^{2}}+u_{y, x}^{p, k^{2}}+u_{z, x}^{p, k^{2}}\right) & \frac{1}{2}\left(u_{x, y}^{p, k^{2}}+u_{y, y}^{p, k^{2}}+u_{z, y}^{p, k^{2}}\right) & u_{x, x}^{p, k} u_{x, y}^{p, k}+u_{y, x}^{p, k} u_{y, y}^{p, k}+u_{z, x}^{p, k} z_{z, y}^{p, k}\end{array}\right\}^{\mathrm{T}}$
$\boldsymbol{\eta}_{\mathrm{n}}^{p, k}=\left\{\begin{array}{lll}u_{x, y}^{p, k} p_{x, z}^{p, k}+u_{y, y}^{p, k} u_{y, z}^{p, k}+u_{z, y}^{p, k} u_{z, z}^{p, k} & u_{x, x}^{p, k} u_{x, z}^{p, k}+u_{y, x}^{p, k} u_{y, z}^{p, k}+u_{z, x}^{p, k} u_{z, z}^{p, k} & u_{x, z}^{p, k} u_{x, z}^{p, k}+u_{y, z}^{p, k} u_{y, z}^{p, k}+u_{z, z}^{p, k} u_{z, z}^{p, k}\end{array}\right\}^{\mathrm{T}}$

components associated with the kinematic assumptions. Note that $N_{u_{r}}^{k}$ denotes the order of the expansions, thus a different number of terms can be used for representing the displacement field along the three directions. This means that, for instance, the in-plane displacement components of a generic sublaminate could be described with an equivalent single layer theory of order 4 , and the out-of-plane displacement with a similar theory of order 2 . In this case, the sublaminate theory would be labeled as $\mathrm{ED}_{442}$. At laminate level, the kinematic theory is specified as a string reporting the approaches adopted for the various sublaminates. For instance, $\mathrm{ED}_{332} / \mathrm{ED}_{554} / \mathrm{ED}_{332}$ denotes a three-sublaminate subdivision, where the first and the third sublaminates are modeled using $\mathrm{ED}_{332}$, while $\mathrm{ED}_{554}$ is adopted for the second one. It is also remarked that each sublaminate can be modeled by assuming, independently, an equivalent single layer or a layerwise description for each displacement component.

\subsection{Strain-displacement relations}

As far as the formulation is developed within a displacementbased approach, it is necessary to introduce the relation between the strain and the displacement components. With the aim of treating the thermal buckling problem, the Green-Lagrange strain tensor is introduced as:

$\epsilon_{i j}=\varepsilon_{i j}+\eta_{i j}$

where distinction is made between the linear and the nonlinear parts $\varepsilon_{i j}$ and $\eta_{i j}$, whose components are:

$\varepsilon_{i j}=\frac{1}{2}\left(u_{i, j}+u_{j, i}\right)$

and

$\eta_{i j}=\frac{1}{2} u_{r, i} u_{r, j}$

Following the standard CUF formalism, the components of Eqs. (4) and (5) are organized in vectors where the in-plane and the normal components are conveniently separated. Considering, for now, the linear part of the strain tensor, it is possible to write: where, consistently with Eq. (6), distinction is made between the in-plane and the normal components.

\subsection{Hooke's law}

The same partitioning into in-plane and normal components of Eq. (6) is adopted, and linear hyperelastic orthotropic behaviour is assumed. After rotating the components into a common laminate reference system, the $3 \mathrm{D}$ constitutive law of the generic ply $p$ of the sublaminate $k$ can be written as:

$\boldsymbol{\sigma}_{\Omega}^{p, k}=\tilde{\mathbf{C}}_{\Omega \Omega}^{p, k} \epsilon_{\Omega}^{p, k}+\tilde{\mathbf{C}}_{\Omega \mathrm{n}}^{p, k} \epsilon_{\mathrm{n}}^{p, k} \quad \boldsymbol{\sigma}_{\mathrm{n}}^{p, k}=\tilde{\mathbf{C}}_{\mathrm{n} \Omega}^{p, k} \epsilon_{\Omega}^{p, k}+\tilde{\mathbf{C}}_{\mathrm{nn}}^{p, k} \epsilon_{\mathrm{n}}^{p, k}$

where:

$\tilde{\mathbf{C}}_{\Omega \Omega}^{p, k}=\left[\begin{array}{ccc}\widetilde{C}_{11}^{p, k} & \widetilde{C}_{12}^{p, k} & \widetilde{C}_{16}^{p, k} \\ \widetilde{C}_{12}^{p, k} & \widetilde{C}_{22}^{p, k} & \widetilde{C}_{26}^{p, k} \\ \widetilde{C}_{16}^{p, k} & \widetilde{C}_{26}^{p, k} & \widetilde{C}_{66}^{p, k}\end{array}\right] \quad \tilde{\mathbf{C}}_{\Omega \mathrm{n}}^{p, k}=\left[\begin{array}{ccc}0 & 0 & \widetilde{C}_{13}^{p, k} \\ 0 & 0 & \widetilde{C}_{23}^{p, k} \\ 0 & 0 & \widetilde{C}_{36}^{p, k}\end{array}\right]$

$\tilde{\mathbf{C}}_{\mathrm{nn}}^{p, k}=\left[\begin{array}{ccc}\widetilde{C}_{44}^{p, k} & \widetilde{C}_{45}^{p, k} & 0 \\ \widetilde{C}_{45}^{p, k} & \widetilde{C}_{55}^{p, k} & 0 \\ 0 & 0 & \widetilde{C}_{33}^{p, k}\end{array}\right]$

Thermal deformations, which are of concern for the prebuckling analysis, are introduced as [2,49]:

${ }_{t} \epsilon_{\Omega}^{p, k}=-\alpha_{\Omega}^{p, k} \Delta T \quad{ }_{t} \epsilon_{n}^{p, k}=-\alpha_{n}^{p, k} \Delta T$

where $\Delta T$ denotes the temperature increase with respect to a reference value, while the vectors collecting the coefficients of thermal expansion are defined as:

$\boldsymbol{\alpha}_{\Omega}^{p, k}=\left\{\begin{array}{lll}\alpha_{x x}^{p, k} & \alpha_{y y}^{p, k} & 2 \alpha_{x y}^{p, k}\end{array}\right\}^{\mathrm{T}} \quad \boldsymbol{\alpha}_{n}^{p, k}=\left\{\begin{array}{lll}0 & 0 & \alpha_{z z}^{p, k}\end{array}\right\}^{\mathrm{T}}$

\subsection{Pre-buckling state}

Following Noor and Burton [28], the pre-buckling analysis is performed by assuming uniform heating and boundary conditions of immovable edges. More specifically, the panel is constrained such that the normal in-plane displacement components are prevented along the four edges. On the other hand, the out-of-plane 
displacement is constrained in correspondence of the midsurface, so that the panel is free to expand or contract along the thickness-wise direction. It follows that the deformed pre-buckling deformed pattern is given by:

$$
\left\{\begin{array}{l}
{ }_{0} u_{x}(x, y, z)={ }_{0} u_{y}(x, y, z)=0 \\
{ }_{0} u_{z}(x, y, z)={ }_{0} u_{z}(z)
\end{array}\right.
$$

where the subscript 0 is introduced to specify the pre-buckling condition. The internal stress distribution arising from the displacement field given by Eq. (14) is:

$$
\left\{\begin{array}{l}
{ }_{0} \sigma_{y z}(x, y, z)={ }_{0} \sigma_{x z}(x, y, z)=0 \\
{ }_{0} \sigma_{\alpha \beta}(x, y, z)={ }_{0} \sigma_{\alpha \beta}(z) \quad \text { with } \alpha, \beta=x, y
\end{array}\right.
$$

meaning that the only not-null pre-buckling stress components are those relative to the panel membrane behaviour. It is worth highlighting that the terms ${ }_{0} \sigma_{\alpha \beta}$ are functions of $z$ and are, in general, different from ply to ply.

For the pre-buckling condition specified by Eqs. (14) and (15), the evaluation of the normal displacement ${ }_{0} u_{z}$ and the in-plane stress components can be performed in a closed-form manner. After noticing that the only not null pre-buckling strain component is ${ }_{0} \epsilon_{z z}^{p, k}$, the internal stresses are available from the thermoelastic constitutive relation:

$$
\begin{aligned}
& { }_{0} \boldsymbol{\sigma}_{\Omega}^{p, k}=-\tilde{\mathbf{C}}_{\Omega \Omega}^{p, k} \boldsymbol{\alpha}_{\Omega}^{p, k} \Delta T+\tilde{\mathbf{C}}_{\Omega \mathrm{n}}^{p, k}\left({ }_{0} \boldsymbol{\epsilon}_{\mathrm{n}}^{p, k}-\boldsymbol{\alpha}_{\mathrm{n}}^{p, k} \Delta T\right) \\
& { }_{0} \boldsymbol{\sigma}_{\mathrm{n}}^{p, k}=-\tilde{\mathbf{C}}_{\mathrm{n} \Omega}^{p, k} \boldsymbol{\alpha}_{\Omega}^{p, k} \Delta T+\tilde{\mathbf{C}}_{\mathrm{nn}}^{p, k}\left({ }_{0} \boldsymbol{\epsilon}_{\mathrm{n}}^{p, k}-\boldsymbol{\alpha}_{\mathrm{n}}^{p, k} \Delta T\right)
\end{aligned}
$$

where:

${ }_{0} \epsilon_{\mathrm{n}}^{p, k}=\left\{\begin{array}{lll}0 & 0 & { }_{0} \epsilon_{z z}^{p, k}\end{array}\right\}^{\mathrm{T}}$

The value of ${ }_{0} \epsilon_{z z}^{p, k}$ can be determined after imposing the vanishing of the normal stress $\sigma_{z z}^{p, k}$, viz. considering plane stress conditions. More specifically, the condition $\sigma_{z z}^{p, k}=0$ can be substituted in Eq. (16), and the pre-buckling strain is obtained as function of the temperature variation as:

$\left.{ }_{0} \epsilon_{z z}^{p, k}={ }_{0} \epsilon_{33}^{p, k}=\frac{\widetilde{C}_{13}^{p, k}}{\widetilde{C}_{33}^{p, k}} \alpha_{x x}^{p, k}+\frac{\widetilde{C}_{23}^{p, k}}{\widetilde{C}_{33}^{p, k}} \alpha_{y y}^{p, k}+2 \frac{\widetilde{C}_{36}^{p, k}}{\widetilde{C}_{33}^{p, k}} \alpha_{x y}^{p, k}+\alpha_{z z}^{p, k}\right) \Delta T$

and the corresponding in-plane stress components are retrieved by making use of Eq. (16), so:

$$
\begin{aligned}
& { }_{0} \boldsymbol{\sigma}_{\Omega}^{p, k}=-\hat{\mathbf{C}}^{p, k} \boldsymbol{\alpha}_{\Omega}^{p, k} \Delta T \\
& { }_{0} \boldsymbol{\sigma}_{\mathrm{n}}^{p, k}=\mathbf{0}
\end{aligned}
$$

where

$$
\hat{\mathbf{C}}^{p, k}=\left[\begin{array}{ccc}
\widehat{C}_{11}^{p, k} & \widehat{C}_{12}^{p, k} & \widehat{C}_{16}^{p, k} \\
\widehat{C}_{12}^{p, k} & \widehat{C}_{22}^{p, k} & \widehat{C}_{26}^{p, k} \\
\widehat{C}_{16}^{p, k} & \widehat{C}_{26}^{p, k} & \widehat{C}_{66}^{p, k}
\end{array}\right]
$$

and

$$
\widehat{C}_{i j}=\widetilde{C}_{i j}^{p, k}-\frac{\widetilde{C}_{i 3}^{p, k} \widetilde{C}_{3 j}^{p, k}}{\widetilde{C}_{33}^{p, k}} \quad i, j=1,2,6
$$

If the effect of pre-buckling deformations is neglected - as it is done in standard linear buckling eigenvalue analysis - no additional results are needed, and the solution given by Eq. (19) suffices for performing the subsequent buckling analysis.

On the other hand, an additional step is needed if the analysis is aimed at accounting for the effect of pre-buckling deformations. In particular, the additional quantity to be evaluated is the first derivative of the pre-buckling displacement ${ }_{0} u_{z, z}$, which is easily obtained after recalling the nonlinear expression of the GreenLagrange strain tensor of Eq. (3) and the solution of the prebuckling problem of Eq. (14) as:
${ }_{0} u_{z, z}^{p, k}=-1+\sqrt{1+2{ }_{0} \varepsilon_{z z}^{p, k}}$

Note that the solution of Eq. (22), despite its simplicity, accounts for the nonlinearity contained in the Green-Lagrange strain tensor. It is also worth observing the presence of the term ${ }_{0} \varepsilon_{z z}^{p, k}$ in Eq. (22), which makes it necessary the adoption of a theory of order higher than zero with respect to the $u_{z}$ component.

\subsection{Buckling condition}

The buckling equations are derived in the context of a variational framework, starting from the expression of the total potential energy of the panel, which is:

$\Pi=\frac{1}{2} \int_{V} \epsilon: \sigma \mathrm{d} V$

where the potential of the external loads is, in the present investigation, identically null as no mechanical loads are prescribed. The strain tensor $\boldsymbol{\epsilon}$ is intended as the contribution restricted to the mechanical deformations, as no strain energy is associated with the thermal deformations.

The first variation of the potential energy allows to derive the nonlinear pre-buckling equilibrium equations, whose closed-form solution is available from Eqs. (16) and (22). The buckling condition can be found referring to the Trefftz criterion [3]:

$\delta\left(\delta^{2} \Pi\right)=0$

According to the stability criterion of Eq. (24), the buckling condition is readily found by recalling Eqs. (3) and (23), and is expressed as:

$\delta\left(\delta^{2} \Pi\right)=\int_{V}\left(\delta \mathbf{e}: \boldsymbol{\sigma}+\lambda \delta \boldsymbol{\eta}:{ }_{0} \boldsymbol{\sigma}\right) \mathrm{d} V=0$

where ${ }_{0} \boldsymbol{\sigma}$ is the pre-buckling stress tensor, $\lambda$ is the buckling multiplier, while $\mathbf{e}$ is:

$\mathbf{e}=\boldsymbol{\varepsilon}+\hat{\boldsymbol{\varepsilon}}$

The components of the tensors $\boldsymbol{\varepsilon}$ and $\boldsymbol{\eta}$ are available from Eqs. (4) and (5), while the term $\hat{\boldsymbol{\varepsilon}}$ is the linear part of the incremental Green-Lagrange strain tensor and its components read:

$\hat{\varepsilon}_{i j}=\frac{1}{2}\left({ }_{0} u_{r, i} u_{r, k}+{ }_{0} u_{r, k} u_{r, i}\right)$

It is highlighted that the components $\hat{\varepsilon}_{i j}$ are identically null whenever pre-buckling deformations are null or negligible. In this case the variational statement of Eq. (25) reduces to the wellknown form:

$\delta\left(\delta^{2} \Pi\right)=\int_{V}\left(\delta \boldsymbol{\varepsilon}: \boldsymbol{\sigma}+\lambda \delta \boldsymbol{\eta}:{ }_{0} \boldsymbol{\sigma}\right) \mathrm{d} V=0$

The more general case of non negligible pre-buckling deformations is now considered. Introducing the S-GUF approximation, and recalling the pre-buckling condition expressed by Eq. (19), the buckling condition of Eq. (25) can be re-written as:

$\sum_{k=1}^{N^{k}} \sum_{p=1}^{N_{p}^{k}} \int_{\Omega} \int_{z_{p}^{\text {bot }}}^{z_{p}^{\text {top }}}\left[\left(\delta \boldsymbol{\varepsilon}^{p, k^{\mathrm{T}}}+\delta \hat{\boldsymbol{\varepsilon}}^{p, k^{\mathrm{T}}}\right) \boldsymbol{\sigma}^{p, k}+\lambda \delta \boldsymbol{\eta}_{\Omega}^{p, k^{\mathrm{T}}}{ }_{0} \boldsymbol{\sigma}_{\Omega}^{p, k}\right] \mathrm{d} z \mathrm{~d} \Omega=0$

where, according to the pre-buckling solution given by Eq. (16), the components of $\hat{\boldsymbol{\varepsilon}}^{p, k}$ are:

$$
\begin{aligned}
\hat{\boldsymbol{\varepsilon}}_{\Omega}^{p, k} & =\left\{\begin{array}{lll}
0 & 0 & 0
\end{array}\right\}^{\mathrm{T}} \\
\hat{\boldsymbol{\varepsilon}}_{\mathrm{n}}^{p, k} & =\left\{\begin{array}{lll}
\hat{\gamma}_{y z}^{p, k} & \hat{\gamma}_{x z}^{p, k} & \hat{\epsilon}_{z z}^{p, k}
\end{array}\right\}^{\mathrm{T}} \\
& =\left\{\begin{array}{lll}
{ }_{0} u_{z, z}^{p, k} u_{z, y}^{p, k} & { }_{0} u_{z, z}^{p, k} u_{z, x}^{p, k} & { }_{0} u_{z, z}^{p, k} u_{z, z}^{p, k}
\end{array}\right\}^{\mathrm{T}}
\end{aligned}
$$


Substituting now Eq. (30) into the variational principle of Eq. (29) leads to:

$\sum_{k=1}^{N^{k}} \sum_{p=1}^{N_{p}^{k}} \int_{\Omega} \int_{z_{p}^{b o t}}^{z_{p}^{\text {top }}}\left[\delta \boldsymbol{\varepsilon}_{\Omega}^{p, k^{\mathrm{T}}} \boldsymbol{\sigma}_{\Omega}^{p, k}+\left(\delta \boldsymbol{\varepsilon}_{\mathrm{n}}^{p, k}+\delta \hat{\boldsymbol{\varepsilon}}_{\mathrm{n}}^{p, k}\right)^{\mathrm{T}} \boldsymbol{\sigma}_{\mathrm{n}}^{p, k}+\lambda \delta \boldsymbol{\eta}_{\Omega}^{p, k^{\mathrm{T}}}{ }_{0} \boldsymbol{\sigma}_{\Omega}^{p, k}\right] \mathrm{d} z \mathrm{~d} \Omega=0$

which is the variational statement expressing the buckling condition in the presence of nonlinear pre-buckling deformations. In the context of a displacement-based formulation, the various terms of Eq. (31) need to be expressed as function of the displacement components. This is done by recalling Eq. (6) and assuming that the constitutive relation can be written in the form:

$\boldsymbol{\sigma}_{\Omega}^{p, k}=\tilde{\mathbf{C}}_{\Omega \Omega}^{p, k} \mathbf{e}_{\Omega}^{p, k}+\tilde{\mathbf{C}}_{\Omega \mathrm{n}}^{p, k} \mathbf{e}_{\mathrm{n}}^{p, k} \quad \boldsymbol{\sigma}_{\mathrm{n}}^{p, k}=\tilde{\mathbf{C}}_{\mathrm{n} \Omega}^{p, k} \mathbf{e}_{\Omega}^{p, k}+\tilde{\mathbf{C}}_{\mathrm{nn}}^{p, k} \mathbf{e}_{\mathrm{n}}^{p, k}$

In addition, the relation between the strains $\hat{\boldsymbol{\varepsilon}}_{\mathrm{n}}^{p, k}$ and the displacement components can be organized by separating the derivatives with respect to the in-plane and the normal components as:

$\hat{\boldsymbol{\varepsilon}}_{\mathrm{n}}^{p, k}={ }_{0} u_{z, z}^{p, k}\left(\hat{\mathbf{D}}_{\mathrm{n}} \mathbf{u}^{p, k}+\hat{\mathbf{D}}_{z} \mathbf{u}^{p, k}\right)$

where the differential matrices $\hat{\mathbf{D}}_{\mathrm{n}}$ and $\hat{\mathbf{D}}_{z}$ are defined as:

$\hat{\mathbf{D}}_{\mathrm{n}}=\left[\begin{array}{ccc}0 & 0 & (\cdot)_{y} \\ 0 & 0 & (\cdot)_{, z} \\ 0 & 0 & 0\end{array}\right] \quad \hat{\mathbf{D}}_{z}=\left[\begin{array}{ccc}0 & 0 & 0 \\ 0 & 0 & 0 \\ 0 & 0 & (\cdot)_{, z}\end{array}\right]$

Substituting now Eqs. (32) and (33) in Eq. (31), it is possible to express the buckling condition as function of the displacement unknowns $\mathbf{u}^{p, k}$. In particular, the three contributions reported in Eq. (31) are written as:

$$
\begin{aligned}
\delta \boldsymbol{\varepsilon}_{\Omega}^{p, k^{\mathrm{T}}} \boldsymbol{\sigma}_{\Omega}^{p, k}= & \delta\left(\mathbf{D}_{\Omega} \mathbf{u}^{p, k}\right)^{\mathrm{T}}\left\{\tilde{\mathbf{C}}_{\Omega \Omega}^{p, k} \mathbf{D}_{\Omega} \mathbf{u}^{p, k}+\tilde{\mathbf{C}}_{\Omega \mathrm{n}}^{p, k}\left[\mathbf{D}_{\mathrm{n}} \mathbf{u}^{p, k}+\mathbf{D}_{z} \mathbf{u}^{p, k}\right.\right. \\
& \left.\left.+{ }_{0} u_{z, z}^{p, k}\left(\hat{\mathbf{D}}_{n} \mathbf{u}^{p, k}+\hat{\mathbf{D}}_{z} \mathbf{u}^{p, k}\right)\right]\right\}
\end{aligned}
$$

$$
\begin{aligned}
\left(\delta \boldsymbol{\varepsilon}_{\mathrm{n}}^{p, k}+\delta \hat{\boldsymbol{\varepsilon}}_{\mathrm{n}}^{p, k}\right)^{\mathrm{T}} \boldsymbol{\sigma}_{\mathrm{n}}^{p, k}= & \delta\left[\mathbf{D}_{n} \mathbf{u}^{p, k}+\mathbf{D}_{z} \mathbf{u}^{p, k}+{ }_{0} u_{z, z}^{p, k}\left(\hat{\mathbf{D}}_{n} \mathbf{u}^{p, k} \hat{\mathbf{D}}_{z} \mathbf{u}^{p, k}\right)\right]^{\mathrm{T}} \\
& \times\left\{\tilde{\mathbf{C}}_{\mathrm{n} \Omega}^{p, k} \mathbf{D}_{\Omega} \mathbf{u}^{p, k}+\tilde{\mathbf{C}}_{\mathrm{nn}}^{p, k}\left[\mathbf{D}_{n} \mathbf{u}^{p, k}+\mathbf{D}_{z} \mathbf{u}^{p, k}\right.\right. \\
& \left.\left.+{ }_{0} u_{z, z}^{p, k}\left(\hat{\mathbf{D}}_{n} \mathbf{u}^{p, k}+\hat{\mathbf{D}}_{z} \mathbf{u}^{p, k}\right)\right]\right\}
\end{aligned}
$$

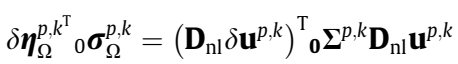

It is noted that all the terms of Eqs. (35) and (36) multiplied with ${ }_{0} u_{z, z}^{p, k}$ are those associated with the nonlinear pre-buckling contributions, while the remaining ones are the standard terms contributing to the stiffness matrix of the undeformed configuration.

The contribution of Eq. (37) is written after introducing the prestress matrix ${ }_{0} \Sigma^{p, k}$ as:

${ }_{0} \boldsymbol{\Sigma}^{p, k}=\left[\begin{array}{ccc}0 \varsigma^{p, k} & 0 & 0 \\ 0 & { }_{0}^{p} \varsigma^{p, k} & 0 \\ 0 & 0 & 0 \varsigma^{p, k}\end{array}\right]$

with

${ }_{0} \varsigma^{p, k}=\left[\begin{array}{cc}{ }_{0} \sigma_{x x}^{p, k} & { }_{0} \sigma_{x y}^{p, k} \\ { }_{0} \sigma_{x y}^{p, k} & { }_{0} \sigma_{y y}^{p, k}\end{array}\right]$

The pre-buckling stress components collected in Eq. (39) are available from Eq. (19) and, in general, are different from ply to ply depending on their thermoelastic properties.

The differential operator $\mathbf{D}_{\mathrm{nl}}$ relative to the nonlinear part of the strain tensor is:
$\mathbf{D}_{\mathrm{nl}}=\left[\begin{array}{ccc}(\cdot)_{, x} & 0 & 0 \\ (\cdot)_{, y} & 0 & 0 \\ 0 & (\cdot)_{, x} & 0 \\ 0 & (\cdot)_{y} & 0 \\ 0 & 0 & (\cdot)_{, x} \\ 0 & 0 & (\cdot)_{y}\end{array}\right]$

Integration along the thickness of Eq. (31) can be performed by recalling the GUF expansion of the displacement unknowns of Eq. (2), which depends upon the theory adopted. In general, the thickness integrals can be written in the form:

$Z_{u_{r} u_{s}}^{p \alpha_{u_{u}} \beta_{u_{s}}}=\int_{z_{p}^{\text {bot }}}^{z_{p}^{\text {top }}} F_{\alpha_{u_{r}}} F_{\beta_{u_{s}}} \mathrm{~d} z \quad Z_{\partial u_{r} u_{s}}^{p \alpha_{u_{r}} \beta_{u_{s}}}=\int_{z_{p}^{\text {bot }}}^{z_{p}^{\text {top }}} F_{\alpha_{u_{r}}, z} F_{\beta_{u_{s}}} \mathrm{~d} z$

$Z_{u_{r} \partial u_{s}}^{p \alpha_{u_{u}} u_{u_{s}}}=\int_{z_{p}^{\mathrm{bot}}}^{z_{p}^{\mathrm{top}}} F_{\alpha_{u_{r}}} F_{\beta_{u_{s}}, z} \mathrm{~d} z \quad Z_{\partial u_{r} \partial u_{s}}^{p \alpha_{u_{r}} \beta_{u_{s}}}=\int_{z_{p}^{\mathrm{bot}}}^{z_{p}^{\mathrm{top}}} F_{\alpha_{u_{r}}, z} F_{\beta_{u_{s}}, z} \mathrm{~d} z$

In the present Ritz-based approach, the in-plane integration of Eq. (31) is performed after approximating the in-plane behaviour by means of properly chosen shape functions.

\section{Ritz approximate solution}

The exact solution of the buckling equations expressed by Eq. (31) can be found for special sets of stacking sequences, loading and boundary conditions. An approximate solution is here sought not to restrict the class of problems to be analyzed, thus making it possible to study different sets of boundary conditions and layups. The solution procedure relies upon a Ritz approximation of the generalized displacement components introduced by Eq. (2) (see also [46,48,37]), which are expressed as:

$\left\{\begin{array}{l}u_{x \alpha_{u_{x}}}^{p, k}(x, y)=N_{u_{x} i}(x, y) u_{x \alpha_{u_{x} i} i}^{p, k} \\ u_{y \alpha_{u_{y}}}^{p, k}(x, y)=N_{u_{y} i}(x, y) u_{y \alpha_{u_{y} i}}^{p, k} \quad i=1,2, \ldots, M \\ u_{z \alpha_{u_{z}}}^{p, k}(x, y)=N_{u_{z} i}(x, y) u_{z \alpha_{u_{z}} i}^{p, k}\end{array}\right.$

where different sets of global shape functions are adopted to approximate the three components $u_{r \alpha_{u_{r}}}^{p, k}(x, y)$. A map is introduced between the physical and the computational domain $(\xi, \eta)$ defined in the interval $\left[\begin{array}{ll}-1 & 1\end{array}\right] \times\left[\begin{array}{ll}-1 & 1\end{array}\right]$, where the generic shape function can be expressed as:

$N_{u_{r} i}(\xi, \eta)=\phi_{u_{r} m}(\xi) \psi_{u_{r} n}(\eta) \quad m=1, \ldots, R \quad n=1, \ldots, S$

and the number of functions $R$ and $S$ along the directions $\xi$ and $\eta$ can be, in general, different. The relation between the generic $i$-th function and the $m$-th and $n$-th one-dimensional functions is given by:

$i=S(m-1)+n$

The functions $\phi_{u_{r} m}(\xi)$ and $\psi_{u_{r} n}(\eta)$ of Eq. (43) are, in turn, expressed as the product between sets of complete functions $p_{r}$ and proper boundary functions, chosen depending on the constraints applied at the panel edges:

$\phi_{u_{r} m}(\xi)=f_{u_{r}}(\xi) p_{m}(\xi)$

$\psi_{u_{r} n}(\eta)=g_{u_{r}}(\eta) p_{n}(\eta)$

In the present implementation, Legendre orthogonal polynomials are adopted as global functions, thus:

$p_{0}=1 ; \quad p_{1}=\zeta ; \quad p_{l+1}=\frac{(2 l+1) \zeta p_{l}-l p_{l-1}}{l+1} \quad$ with $l=m, n$

while the boundary functions are the polynomial expressions reported in Ref. [46]. 
For comparison purposes, a second set of Ritz functions, taken from Ref. [39], is considered. In particular, the set consists of the trigonometric functions defined as:

$$
\begin{aligned}
& N_{u_{x} i}(\xi, \eta)=\cos \frac{m \pi}{2}(\xi+1) \sin \frac{n \pi}{2}(\eta+1) \\
& N_{u_{y} i}(\xi, \eta)=\sin \frac{m \pi}{2}(\xi+1) \cos \frac{n \pi}{2}(\eta+1) \\
& N_{u_{z} i}(\xi, \eta)=\sin \frac{m \pi}{2}(\xi+1) \sin \frac{n \pi}{2}(\eta+1)
\end{aligned}
$$

It should be highlighted that the functions of Eq. (47) are suitable for analyzing simply-supported boundary conditions, while they cannot be employed to analyze other set of boundary conditions.

Independently on the choice for the Ritz functions, the approximation of Eq. (43) can be substituted into the expression of Eq. (31) and, after evaluating the surface integrals and assembling the contributions at ply and sublaminate level [36,37], the governing equations are obtained in the form:

$\delta \mathbf{u}^{\mathrm{T}}\left[\left(\mathbf{K}+\mathbf{K}^{0}\right)+\lambda \mathbf{K}^{\mathrm{G}}\right] \mathbf{u}=\mathbf{0} \quad \forall \delta \mathbf{u}$

where $\mathbf{K}$ is the stiffness matrix, $\mathbf{K}^{0}$ is the contribution due the prebuckling deformations and $\mathbf{K}^{\mathrm{G}}$ is the geometric stiffness. The vector of unknowns is given by:

$\mathbf{u}=\left\{\mathbf{u}_{1} \cdots \mathbf{u}_{R S}\right\}^{\mathrm{T}}$

and the $i$-th contribution appearing in Eq. (49) is the set of unknown amplitudes associated with the $i$-th shape function, partitioned into the $x, y$ and $z$-wise contributions as:

$\mathbf{u}_{i}=\left\{\begin{array}{lll}\mathbf{u}_{x i} & \mathbf{u}_{y i} & \mathbf{u}_{z i}\end{array}\right\}^{\mathrm{T}}$

The expression of the stiffness matrix $\mathbf{K}$ is already available in Ref. [37] but, for completeness, its expression is summarized in the Appendix.

The stiffness contribution associated with the pre-buckling deformations is obtained as:

$\mathbf{K}^{0}=\mathbf{K}_{u_{x} u_{z}}^{0}+\mathbf{K}_{u_{x} u_{z}}^{0^{\mathrm{T}}}+\mathbf{K}_{u_{y} u_{z}}^{0}+\mathbf{K}_{u_{y} u_{z}}^{0^{\mathrm{T}}}+\mathbf{K}_{u_{z} u_{z}}^{0}$

where the single terms of Eq. (51) are organized to highlight the work-conjugacy dependence over the different displacement components $u_{r}$. The formulation is developed by keeping separate the thickness-wise and the in-plane response, as clear from Eq. (2). It follows that the various terms composing the stiffness matrices can be obtained by composing two distinct contributions: those relative to the integration along the thickness, and those relative to inplane integration of Eq. (31). Referring to Eq. (51), the three distinct contributions are obtained by composing the assembled thickness integrals $\mathbf{Z}_{(\partial) u_{r} \partial(\partial) u_{S} R S}^{0}$, whose expression is reported in the Appendix, and the in-plane integrals as:

$\mathbf{K}_{u_{x} u_{z}}^{0}=\mathcal{I}_{u_{x} u_{z}}^{0100} \otimes \mathbf{Z}_{u_{x} \partial u_{z} 36}^{0}+\mathcal{I}_{u_{x} u_{z}}^{0010} \otimes \mathbf{Z}_{\partial u_{x} u_{z} 55}^{0}+\mathcal{I}_{u_{x} u_{z}}^{1000} \otimes \mathbf{Z}_{u_{x} \partial u_{z} 13}^{0}+\mathcal{I}_{u_{x} u_{z}}^{0001} \otimes \mathbf{Z}_{\partial u_{x} u_{z} 45}^{0}$

$\mathbf{K}_{u_{y} u_{z}}^{0}=\mathcal{I}_{u_{y} u_{z}}^{0010} \otimes \mathbf{Z}_{\partial u_{y} u_{z} 45}^{0}+\mathcal{I}_{u_{y} u_{z}}^{0100} \otimes \mathbf{Z}_{u_{y} \partial u_{z} 23}^{0}+\mathcal{I}_{u_{y} u_{z}}^{0001} \otimes \mathbf{Z}_{\partial u_{y} u_{z} 44}^{0}+\mathcal{I}_{u_{y} u_{z}}^{1000} \otimes \mathbf{Z}_{u_{y} \partial u_{z} 36}^{0}$

$\mathbf{K}_{u_{z} u_{z}}^{0}=\mathcal{I}_{u_{z} u_{z}}^{0101} \otimes \mathbf{Z}_{u_{z} u_{z} 44}^{0}+\mathcal{I}_{u_{z} u_{z}}^{0000} \otimes \mathbf{Z}_{\partial u_{z} \partial u_{z} 33}^{0}+\left(\mathcal{I}_{u_{z} u_{z}}^{0110}+\mathcal{I}_{u_{z} u_{z}}^{1001}\right) \otimes \mathbf{Z}_{u_{z} u_{z} 45}^{0}$

$+\mathcal{I}_{u_{z} u_{z}}^{1010} \otimes \mathbf{Z}_{u_{z} u_{z} 55}^{0}$

where $\otimes$ denotes the Kronecker product and $\mathcal{I}_{u_{r} u_{s}}^{\text {defg }}$ are the matrices collecting the integrals of the Ritz functions according to the following notation:

$\left(\mathcal{I}_{u_{r} u_{s}}^{\text {defg }}\right)_{i j}=\int_{-1}^{1} \int_{-1}^{1} \frac{\partial^{d+e} N_{u_{r} i}}{\partial \xi^{d} \partial \eta^{e}} \frac{\partial^{f+g} N_{u_{s} j}}{\partial \xi^{f} \partial \eta^{g}} \mathrm{~d} \eta \mathrm{d} \xi \quad(d, e, f, g=0,1)$
In a similar fashion, the geometric stiffness matrix is obtained as:

$\mathbf{K}^{\mathrm{G}}=\mathbf{K}_{u_{x} u_{x}}^{\mathrm{G}}+\mathbf{K}_{u_{y} u_{y}}^{\mathrm{G}}+\mathbf{K}_{u_{z} u_{z}}^{\mathrm{G}}$

where:

$\mathbf{K}_{u_{x} u_{x}}^{G}=\mathcal{I}_{u_{x} u_{x}}^{1010} \otimes \mathbf{Z}_{u_{x} u_{x} 11}^{G}+\mathcal{I}_{u_{x} u_{x}}^{0101} \otimes \mathbf{Z}_{u_{x} u_{x} 22}^{G}+\left(\mathcal{I}_{u_{x} u_{x}}^{1001}+\mathcal{I}_{u_{x} u_{x}}^{0110}\right) \otimes \mathbf{Z}_{u_{x} u_{x} 12}^{G}$

$\mathbf{K}_{u_{y} u_{y}}^{G}=\mathcal{I}_{u_{y} u_{y}}^{1010} \otimes \mathbf{Z}_{u_{y} u_{y} 11}^{G}+\mathcal{I}_{u_{y} u_{y}}^{0101} \otimes \mathbf{Z}_{u_{y} u_{y} 22}^{G}+\left(\mathcal{I}_{u_{y} u_{y}}^{1001}+\mathcal{I}_{u_{y} u_{y}}^{0110}\right) \otimes \mathbf{Z}_{u_{y} u_{y} 12}^{G}$

$\mathbf{K}_{u_{z} u_{z}}^{G}=\mathcal{I}_{u_{z} u_{z}}^{1010} \otimes \mathbf{Z}_{u_{z} u_{z} 11}^{G}+\mathcal{I}_{u_{z} u_{z}}^{0101} \otimes \mathbf{Z}_{u_{z} u_{z} 22}^{G}+\left(\mathcal{I}_{u_{z} u_{z}}^{1001}+\mathcal{I}_{u_{z} u z}^{0110}\right) \otimes \mathbf{Z}_{u_{z} u_{z} 12}^{G}$

and the generic contribution $\mathbf{Z}_{u_{r} u_{s} R S}^{\mathrm{G}}$ is reported in the appendix.

The solution of the buckling problem of Eq. (48) is performed by implementing an iterative procedure. Firstly, the eigenvalue problem is solved by neglecting the pre-buckling deformations, i.e. setting $\mathbf{K}^{0}$ to a zero matrix, thus reducing to the standard form of the buckling eigenvalue problem. Then, the pre-buckling state is updated by using Eqs. (18), (19) and (22), and, accordingly, the matrices $\mathbf{K}^{0}$ and $\mathbf{K}^{\mathrm{G}}$ of Eqs. (51) and (54) are re-computed. The eigenvalue problem of Eq. (48) is then solved again, and the procedure is repeated until convergence between two successive iterations is reached. Classical buckling solutions are obtained by neglecting the pre-buckling deformations, i.e. arresting the procedure at the first iteration.

\section{Results}

This section presents the thermal buckling results obtained for monolithic and sandwich plates, derived with and without accounting for the effects of nonlinear pre-buckling deformations. It is highlighted that one single sublaminate is adopted for analyzing monolithic plates and, in that case, the kinematic models reduce to those formulated by the standard GUF approach.

Comparisons are discussed against reference solutions available in the literature, and additional results are provided for future comparison purposes.

The thermoelastic properties of the materials considered throughout the study are summarized in Table 1 . The labels M1 to M3 refer to typical fibrous composite materials.

For sandwich panels, the face-sheets are assumed to be made of material M3, while the core is labeled as $\mathrm{C} 1$. The properties of the material C1 are taken from Ref. [26] and, for convenience, are summarized here below:

- $E_{11} / E_{22, \mathrm{M} 3}=3.2 \mathrm{e}-5 ; E_{22} / E_{22, \mathrm{M} 3}=2.9 \mathrm{e}-5 ; E_{33} / E_{22, \mathrm{M} 3}=0.4$

- $G_{12} / E_{22, \mathrm{M} 3}=2.4 \mathrm{e}-3 ; G_{13} / E_{22, \mathrm{M} 3}=7.9 \mathrm{e}-2 ; G_{23} / E_{22, \mathrm{M} 3}=6.6 \mathrm{e}-2$

- $v_{12}=0.99 ; v_{13}=v_{23}=3.0 \mathrm{e}-5$;

- $\alpha_{11} / \alpha_{0}=\alpha_{22} / \alpha_{0}=\alpha_{33} / \alpha_{0}=1.36$

Table 1

Elastic properties and coefficients of thermal expansion of the materials used in the present study.

\begin{tabular}{llll}
\hline & M1 (Ref. [28]) & M2 (Ref. [39]) & M3 (Ref. [26]) \\
\hline $\mathrm{E}_{11} / \mathrm{E}_{22}$ & 15 & Variable & 19 \\
$\mathrm{E}_{33} / \mathrm{E}_{22}$ & 1 & 1 & 1 \\
$\mathrm{G}_{12} / \mathrm{E}_{22}$ & 0.5 & 0.5310 & 0.52 \\
$\mathrm{G}_{13} / \mathrm{E}_{22}$ & 0.5 & 0.5310 & 0.52 \\
$\mathrm{G}_{23} / \mathrm{E}_{22}$ & 0.3356 & 0.1593 & 0.338 \\
$v_{12}$ & 0.3 & 0.3 & 0.32 \\
$v_{13}$ & 0.3 & 0.3 & 0.32 \\
$v_{23}$ & 0.49 & 0.36 & 0.49 \\
$\alpha_{11} / \alpha_{0}$ & 0.015 & -0.0333 & 0.001 \\
$\alpha_{22} / \alpha_{0}$ & 1 & 1 & 1 \\
$\alpha_{33} / \alpha_{0}$ & 1 & 1 & 1 \\
\hline
\end{tabular}


where $E_{22, \mathrm{M} 3}$ denotes the transverse elastic modulus of the material M3.

It is worth noting that the critical temperatures will be presented, in most cases, in nondimensional form as function of $\alpha_{0} T_{\mathrm{cr}}$. As such, the results do not depend on $E_{22}$ and $\alpha_{0}$, whose values can be arbitrarily fixed; the remaining thermoelastic properties are then recovered by making use of the nondimensional ratios of Table 1 and those of material C1. On the contrary, the dimensional critical temperature $T_{\mathrm{cr}}$ depends on the value of $\alpha_{0}$. This latter will be provided in the next sections whenever the results are presented in dimensional form.

\subsection{Monolithic plates}

\subsubsection{Convergence analysis}

A preliminary convergence analysis is presented to illustrate properties of different shape functions, both in terms of type and number, and to motivate their choice throughout the study. The analysis regards a set of square, anti-symmetrically layered plates, characterized by a length-to-thickness ratio equal to 10 . The plates are obtained by the stacking of 10 plies, oriented at $[ \pm \theta]_{5}$, and each layer is made of the material M1 of Table 1. Simply-supported conditions are imposed at the four edges.

The convergence analysis is performed by considering one single sublaminate and an equivalent single layer theory $\mathrm{ED}_{554}$. Note that models with $N_{u_{z}}=N_{u_{\alpha}}-1$ and $(\alpha=x, y)$ yield a consistent polynomial approximation of the transverse shear strain $\gamma_{\alpha z}$ of order $N_{u_{z}}$. This approximation is justified by the fact that the investigated composite plates all show a bending-dominated buckling mode, for which the transverse shear strains are known to have a certain relevance.

Both Legendre polynomials and trigonometric functions are used to approximate the generalized displacement components according to Eqs. (46) and (47). The results are computed by varying the number of Ritz functions from $2 \times 2$ until $30 \times 30$. The nondimensional critical temperatures $\alpha_{0} T_{\text {cr }}$ are summarized in Table 2 . Here and henceforth, the acronyms $\mathrm{L}$ and $\mathrm{NL}$ denote the results of the linear and nonlinear buckling analysis, respectively. The comparison against the 3D approximate solutions derived by Noor and Burton [28] is reported to illustrate the accuracy of the solutions here obtained.

The results of Table 2 demonstrate the superior convergence properties of the Legendre polynomials over the trigonometric functions. This conclusion holds both for the linear and nonlinear predictions. The only exception is given by the plates with $\theta$ equal to 0 , for which the trigonometric Ritz functions are capable of rep- resenting the exact solution of the problem (or, alternatively, a Navier-type solution of this configuration can be obtained). It follows that two functions suffice for capturing the exact solution, and clearly no improvements are obtained as the number of degrees of freedom is increased. On the other hand, poor-quality predictions are obtained if the same number of functions is taken by considering Legendre polynomials. In any case, it can be observed that convergence to the exact solution is quickly achieved up to four digits when 10 Legendre polynomials are taken along the two directions.

In all the other cases, Legendre functions guarantee faster convergence. In particular, for $\theta \neq 0$, the buckled pattern is affected by the elastic coupling arising from the anti-symmetry of the laminate. The three displacement components of the first buckling mode are reported in Fig. 2 for $\theta$ equal to 45 . While the out-ofplane displacement is characterized by a square half-wave, the skewness of the two in-plane displacement components can be clearly observed. As it turns out, this buckled pattern is not properly captured using few trigonometric functions, and, for this reason, more terms are needed to reach convergence. On the contrary, Legendre polynomials guarantee a far better approximation and convergence is achieved with 15 terms.

From Table 2 it is clear that, for all the configurations, no improvements are achieved when the number of Legendre functions is increased beyond 15 . On the contrary, the critical temperatures obtained with trigonometric functions, excluding the special case of $\theta=0$, have not reached convergence of the fourth digit even when 30 functions are adopted.

The comparison against the solutions reported by Noor and Burton demonstrates the accuracy of the present formulation. In some cases, the results here obtained are smaller in comparison to Ref. [28]. These latter are obtained by a 3D, yet approximate, solution procedure.

It is worth noting that the orthogonality properties of the Legendre functions guarantee a higher degree of sparsity of the resulting matrices with respect to the trigonometric case. It follows that, for the same number of Ritz functions, the computational effort to solve the eigenvalue problem is much smaller. It is finally remarked that the authors did not experience any sort of numerical issue, both in the case of Legendre as well as trigonometric functions, by increasing the number of shape functions, even much beyond the values indicated in Table 2.

\subsubsection{Example 1}

A comprehensive set of results, useful for benchmarking purposes, is available in Ref. [28]. The test case consists in a set of

Table 2

Convergence analysis and comparison of critical temperatures $\alpha_{0} T_{\text {cr }}$ for square composite SSSS plate of material M1, lay-up $[ \pm \theta]_{5}, a / h=10$ and theory ED 554

\begin{tabular}{|c|c|c|c|c|c|c|c|c|c|}
\hline \multirow[b]{2}{*}{ Analysis type } & \multirow{2}{*}{$\begin{array}{l}\theta \\
\mathrm{R}\end{array}$} & 0 & 15 & 30 & 45 & 0 & 15 & 30 & 45 \\
\hline & & \multicolumn{4}{|c|}{ Trigonometric functions } & \multicolumn{4}{|c|}{ Legendre functions } \\
\hline \multirow[t]{7}{*}{$\mathrm{L}$} & 2 & $0.5782^{-1}$ & $0.8020^{-1}$ & 0.1128 & 0.1231 & $0.8782^{-1}$ & $0.9969^{-1}$ & 0.1210 & 0.1305 \\
\hline & 5 & $0.5782^{-1}$ & $0.7968^{-1}$ & 0.1124 & 0.1226 & $0.5797^{-1}$ & $0.7909^{-1}$ & 0.1111 & 0.1211 \\
\hline & 10 & $0.5782^{-1}$ & $0.7948^{-1}$ & 0.1119 & 0.1221 & $0.5782^{-1}$ & $0.7878^{-1}$ & 0.1106 & 0.1204 \\
\hline & 15 & $0.5782^{-1}$ & $0.7939^{-1}$ & 0.1117 & 0.1218 & $0.5782^{-1}$ & $0.7877^{-1}$ & 0.1105 & 0.1202 \\
\hline & 20 & $0.5782^{-1}$ & $0.7934^{-1}$ & 0.1116 & 0.1216 & $0.5782^{-1}$ & $0.7876^{-1}$ & 0.1105 & 0.1202 \\
\hline & 25 & $0.5782^{-1}$ & $0.7930^{-1}$ & 0.1114 & 0.1214 & $0.5782^{-1}$ & $0.7876^{-1}$ & 0.1105 & 0.1202 \\
\hline & 30 & $0.5782^{-1}$ & $0.7927^{-1}$ & 0.1114 & 0.1213 & $0.5782^{-1}$ & $0.7876^{-1}$ & 0.1105 & 0.1202 \\
\hline 3D, Ref. [28] & $0.5782^{-1}$ & $0.7904^{-1}$ & 0.1100 & 0.1194 & & $0.5782^{-1}$ & $0.7904^{-1}$ & 0.1100 & 0.1194 \\
\hline \multirow[t]{7}{*}{$\mathrm{NL}$} & 2 & $0.6944^{-1}$ & 0.1043 & 0.1688 & 0.1936 & $1.1852^{-1}$ & 0.1411 & 0.1882 & 0.2121 \\
\hline & 5 & $0.6944^{-1}$ & 0.1035 & 0.1679 & 0.1924 & $0.6966^{-1}$ & 0.1025 & 0.1651 & 0.1887 \\
\hline & 10 & $0.6944^{-1}$ & 0.1031 & 0.1669 & 0.1910 & $0.6944^{-1}$ & 0.1019 & 0.1639 & 0.1868 \\
\hline & 15 & $0.6944^{-1}$ & 0.1030 & 0.1664 & 0.1903 & $0.6944^{-1}$ & 0.1019 & 0.1637 & 0.1864 \\
\hline & 20 & $0.6944^{-1}$ & 0.1029 & 0.1661 & 0.1898 & $0.6944^{-1}$ & 0.1019 & 0.1637 & 0.1864 \\
\hline & 25 & $0.6944^{-1}$ & 0.1028 & 0.1659 & 0.1894 & $0.6944^{-1}$ & 0.1019 & 0.1637 & 0.1864 \\
\hline & 30 & $0.6944^{-1}$ & 0.1028 & 0.1657 & 0.1892 & $0.6944^{-1}$ & 0.1019 & 0.1637 & 0.1864 \\
\hline 3D, Ref. [28] & & $0.6950^{-1}$ & 0.1027 & 0.1633 & 0.1849 & $0.6950^{-1}$ & 0.1027 & 0.1633 & 0.1849 \\
\hline
\end{tabular}




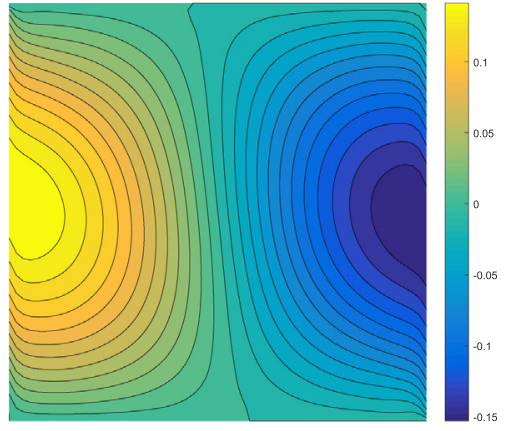

(a)

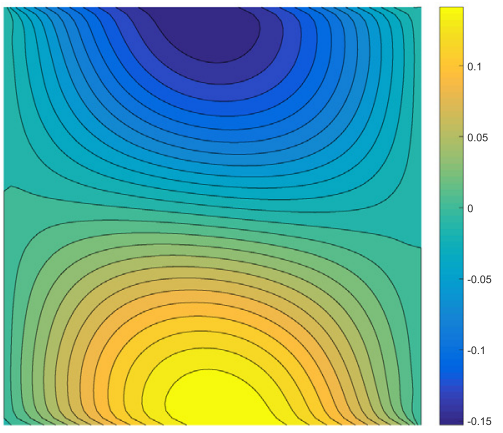

(b)

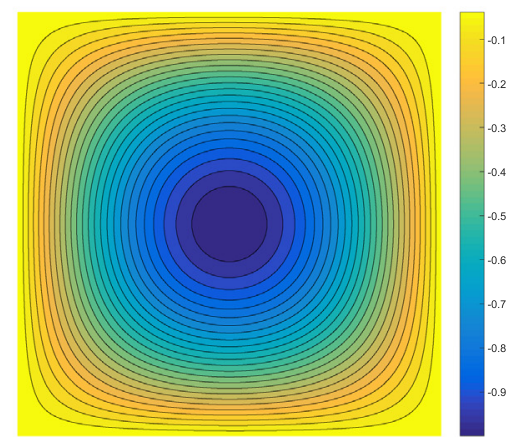

(c)

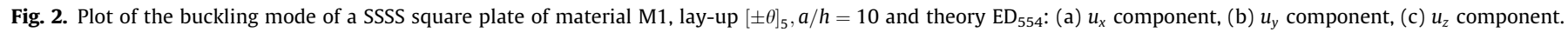

angle-ply laminates and one isotropic configuration, characterized by different nondimensional values of $a / h$. The composite panels are made of material M1 and the lay-up is $[ \pm \theta]_{5}$; the isotropic configurations have a Poisson's coefficient $v=0.3$ and $\alpha_{i i} / \alpha_{0}=1$ (no summation is implied for the index $i$ ). Boundary conditions of simply supported edges are assumed at the four edges of the plate. The results are summarized in Table 3 by adopting $20 \times 20$ Legendre functions and four different kinematic theories.

Note that a few configurations of thick panels with $a / h$ equal to $10 / 3,4$ and 5 and ply angles $\theta$ equal to 30 and 45 are not reported in the table as no reference results are available.

The excellent agreement between the results obtained using the present Ritz formulation can be seen from Table 3. As expected, the critical temperatures diminish as the equivalent single layer description is enriched from $\mathrm{ED}_{332}$ to $\mathrm{ED}_{554}$. Similarly, a reduction of the buckling multiplier is observed by refining the layerwise theory from $L_{111}$ to $L_{222}$. Note that the critical temperatures obtained using $\mathrm{LD}_{111}$ (33 DOF) are, in general, sensibly higher than those predicted by the $\mathrm{LD}_{222}$ model (63 DOF) and, in many cases, by the $\mathrm{ED}_{554}$ model (17 DOF). The reason is attributed to the Poisson locking problem that comes along a linear assumption for the transverse deflection and the associated constant transverse normal strain, see also [50].

As seen from Table 3, the gain in terms of accuracy is modest if the theory is refined from $\mathrm{ED}_{332}$ to the more expensive $\mathrm{LD}_{222}$, even for studying thick panels. Very accurate results are available already from theory $\mathrm{ED}_{332}$, both in terms of linear and nonlinear predictions.

Table 3

Comparison of critical temperatures $\alpha_{0} T_{\text {cr }}$ for square SSSS plates of material M1, lay-up $[ \pm \theta]_{5}$.

\begin{tabular}{|c|c|c|c|c|c|c|c|c|c|c|c|}
\hline \multirow[t]{2}{*}{$a / h$} & \multirow[t]{2}{*}{$\theta$} & \multicolumn{2}{|l|}{ Ref. [28] } & \multicolumn{2}{|l|}{$\mathrm{ED}_{332}$} & \multicolumn{2}{|l|}{$\mathrm{ED}_{554}$} & \multicolumn{2}{|l|}{$\mathrm{LD}_{111}$} & \multicolumn{2}{|l|}{$\mathrm{LD}_{222}$} \\
\hline & & $\mathrm{L}$ & $\mathrm{NL}$ & $\mathrm{L}$ & $\mathrm{NL}$ & $\mathrm{L}$ & $\mathrm{NL}$ & $\mathrm{L}$ & $\mathrm{NL}$ & $\mathrm{L}$ & $\mathrm{NL}$ \\
\hline \multirow[t]{3}{*}{$10 / 3$} & 0 & 0.2057 & 0.4264 & 0.2066 & 0.4266 & 0.2057 & 0.4241 & 0.2069 & 0.4280 & 0.2057 & 0.4241 \\
\hline & 15 & 0.2347 & 0.5719 & 0.2378 & 0.5799 & 0.2354 & 0.5685 & 0.2348 & 0.5653 & 0.2318 & 0.5490 \\
\hline & Isotropic & $0.7193^{-1}$ & $0.9511^{-1}$ & $0.7199^{-1}$ & $0.9514^{-1}$ & $0.7193^{-1}$ & $0.9504^{-1}$ & $0.7219^{-1}$ & $0.9548^{-1}$ & $0.7193^{-1}$ & $0.9504^{-1}$ \\
\hline \multirow[t]{3}{*}{4} & 0 & 0.1777 & 0.3314 & 0.1781 & 0.3303 & 0.1777 & 0.3290 & 0.1786 & 0.3318 & 0.1777 & 0.3290 \\
\hline & 15 & 0.2087 & 0.4598 & 0.2107 & 0.4623 & 0.2090 & 0.4554 & 0.2085 & 0.4530 & 0.2058 & 0.4406 \\
\hline & Isotropic & $0.5600^{-1}$ & $0.6944^{-1}$ & $0.5603^{-1}$ & $0.6939^{-1}$ & $0.5600^{-1}$ & $0.6935^{-1}$ & $0.5618^{-1}$ & $0.6963^{-1}$ & $0.5600^{-1}$ & $0.6935^{-1}$ \\
\hline \multirow[t]{4}{*}{5} & 0 & 0.1436 & 0.2350 & 0.1438 & 0.2348 & 0.1436 & 0.2343 & 0.1442 & 0.2360 & 0.1436 & 0.2343 \\
\hline & 15 & 0.1753 & 0.3352 & 0.1763 & 0.3366 & 0.1753 & 0.3331 & 0.1748 & 0.3316 & 0.1726 & 0.3234 \\
\hline & 30 & 0.2377 & 0.6642 & 0.2441 & 0.6968 & 0.2419 & 0.6812 & 0.2379 & 0.6557 & 0.2344 & 0.6297 \\
\hline & Isotropic & $0.3990^{-1}$ & $0.4644^{-1}$ & $0.3991^{-1}$ & $0.4643^{-1}$ & $0.3990^{-1}$ & $0.4642^{-1}$ & $0.4002^{-1}$ & $0.4658^{-1}$ & $0.3990^{-1}$ & $0.4642^{-1}$ \\
\hline \multirow[t]{5}{*}{$20 / 3$} & 0 & 0.1029 & 0.1448 & 0.1030 & 0.1447 & 0.1029 & 0.1446 & 0.1033 & 0.1454 & 0.1029 & 0.1446 \\
\hline & 15 & 0.1322 & 0.2109 & 0.1325 & 0.2109 & 0.1320 & 0.2096 & 0.1317 & 0.2089 & 0.1300 & 0.2048 \\
\hline & 30 & 0.1859 & 0.4068 & 0.1887 & 0.4165 & 0.1874 & 0.4099 & 0.1849 & 0.3987 & 0.1809 & 0.3778 \\
\hline & 45 & 0.1981 & 0.4713 & 0.2024 & 0.4877 & 0.2006 & 0.4774 & 0.1969 & 0.4576 & 0.1921 & 0.4310 \\
\hline & Isotropic & $0.2468^{-1}$ & $0.2709^{-1}$ & $0.2468^{-1}$ & $0.2707^{-1}$ & $0.2468^{-1}$ & $0.2707^{-1}$ & $0.2475^{-1}$ & $0.2715^{-1}$ & $0.2468^{-1}$ & $0.2707^{-1}$ \\
\hline \multirow[t]{5}{*}{10} & 0 & $0.5782^{-1}$ & $0.6950^{-1}$ & $0.5782^{-1}$ & $0.6946^{-1}$ & $0.5782^{-1}$ & $0.6944^{-1}$ & $0.5800^{-1}$ & $0.6971^{-1}$ & $0.5782^{-1}$ & $0.6945^{-1}$ \\
\hline & 15 & $0.7904^{-1}$ & 0.1027 & $0.7898^{-1}$ & 0.1023 & $0.7876^{-1}$ & 0.1019 & $0.7868^{-1}$ & 0.1018 & $0.7784^{-1}$ & 0.1004 \\
\hline & 30 & 0.1100 & 0.1633 & 0.1110 & 0.1649 & 0.1105 & 0.1637 & 0.1095 & 0.1617 & 0.1076 & 0.1575 \\
\hline & 45 & 0.1194 & 0.1849 & 0.1209 & 0.1882 & 0.1202 & 0.1864 & 0.1187 & 0.1822 & 0.1163 & 0.1766 \\
\hline & Isotropic & $0.1183^{-1}$ & $0.1237^{-1}$ & $0.1183^{-1}$ & $0.1237^{-1}$ & $0.1183^{-1}$ & $0.1237^{-1}$ & $0.1186^{-1}$ & $0.1240^{-1}$ & $0.1183^{-1}$ & $0.1237^{-1}$ \\
\hline \multirow[t]{5}{*}{20} & 0 & $0.1739^{-1}$ & $0.1834^{-1}$ & $0.1739^{-1}$ & $0.1834^{-1}$ & $0.1739^{-1}$ & $0.1834^{-1}$ & $0.1743^{-1}$ & $0.1838^{-1}$ & $0.1739^{-1}$ & $0.1834^{-1}$ \\
\hline & 15 & $0.2528^{-1}$ & $0.2734^{-1}$ & $0.2523^{-1}$ & $0.2727^{-1}$ & $0.2519^{-1}$ & $0.2723^{-1}$ & $0.2520^{-1}$ & $0.2723^{-1}$ & $0.2503^{-1}$ & $0.2704^{-1}$ \\
\hline & 30 & $0.3446^{-1}$ & $0.3843^{-1}$ & $0.3468^{-1}$ & $0.3866^{-1}$ & $0.3459^{-1}$ & $0.3855^{-1}$ & $0.3448^{-1}$ & $0.3842^{-1}$ & $0.3416^{-1}$ & $0.3802^{-1}$ \\
\hline & 45 & $0.3810^{-1}$ & $0.4302^{-1}$ & $0.3839^{-1}$ & $0.4338^{-1}$ & $0.3826^{-1}$ & $0.4322^{-1}$ & $0.3809^{-1}$ & $0.4300^{-1}$ & $0.3767^{-1}$ & $0.4246^{-1}$ \\
\hline & Isotropic & $0.3109^{-2}$ & $0.3145^{-2}$ & $0.3109^{-2}$ & $0.3145^{-2}$ & $0.3109^{-2}$ & $0.3145^{-2}$ & $0.3116^{-2}$ & $0.3153^{-2}$ & $0.3109^{-2}$ & $0.3145^{-2}$ \\
\hline \multirow[t]{5}{*}{100} & 0 & $0.7463^{-3}$ & $0.7480^{-3}$ & $0.7463^{-3}$ & $0.7480^{-3}$ & $0.7463^{-3}$ & $0.7480^{-3}$ & $0.7478^{-3}$ & $0.7495^{-3}$ & $0.7463^{-3}$ & $0.7480^{-3}$ \\
\hline & 15 & $0.1115^{-2}$ & $0.1118^{-2}$ & $0.1114^{-2}$ & $0.1118^{-2}$ & $0.1114^{-2}$ & $0.1117^{-2}$ & $0.1115^{-2}$ & $0.1119^{-2}$ & $0.1113^{-2}$ & $0.1117^{-2}$ \\
\hline & 30 & $0.1502^{-2}$ & $0.1509^{-2}$ & $0.1514^{-2}$ & $0.1521^{-2}$ & $0.1514^{-2}$ & $0.1521^{-2}$ & $0.1514^{-2}$ & $0.1521^{-2}$ & $0.1512^{-2}$ & $0.1519^{-2}$ \\
\hline & 45 & $0.1674^{-2}$ & $0.1683^{-2}$ & $0.1690^{-2}$ & $0.1698^{-2}$ & $0.1689^{-2}$ & $0.1698^{-2}$ & $0.1689^{-2}$ & $0.1698^{-2}$ & $0.1687^{-2}$ & $0.1696^{-2}$ \\
\hline & Isotropic & $0.1264^{-3}$ & $0.1265^{-3}$ & $0.1264^{-3}$ & $0.1265^{-3}$ & $0.1264^{-3}$ & $0.1265^{-3}$ & $0.1267^{-3}$ & $0.1268^{-3}$ & $0.1264^{-3}$ & $0.1265^{-3}$ \\
\hline
\end{tabular}


In agreement with Noor and Burton, the ratio between the linear and the nonlinear critical temperature diminishes as the ratio $a / h$ increases, and, for thin plates, these two values are almost identical. On the contrary, a noticeable difference can be observed between the linear and nonlinear predictions, especially for thick configurations, with a difference between the values that increases with the anisotropy of the lay-up. Isotropic plates are, in all the cases, those characterized by the closer values between linear and nonlinear results, whilst laminates with $\theta$ equal to 45 are those exhibiting the highest differences.

A plot of the nondimensional parameter $a / h$ versus the critical temperature ratio is provided in Fig. 3. This latest quantity is defined as the ratio between the linear buckling prediction and the nonlinear one, thus providing a measure of the effects due to the pre-buckling deformations. The close agreement with the results extracted from Ref. [28] can be seen even in this case.

\subsubsection{Example 2}

Another test case is taken from Ref. [39]. Relatively thin plates with $a / h$ equal to 50 are considered. The plates are made of material M2 and obtained by the stacking of four plies oriented at $[ \pm \theta]_{s}$. Simply-supported boundary conditions are imposed at the plate edges. The results are summarized in Table 4 in terms of dimensional critical temperatures, obtained by taking $E_{11} / E_{22}=11.292$, and $\alpha_{0}=2710^{-6} / \mathrm{K}$.

The results taken from Ref. [39] are obtained using the CUFbased $\mathrm{ED}_{222}$ theory in conjunction with a Ritz approach employing $12 \times 12$ trigonometric shape functions. For consistency, present results are reported in the adjacent column and denoted $E D_{222 a}$, which are obtained using the same model and Ritz function as in Ref. [39]: as expected, the results are identical. The remaining set

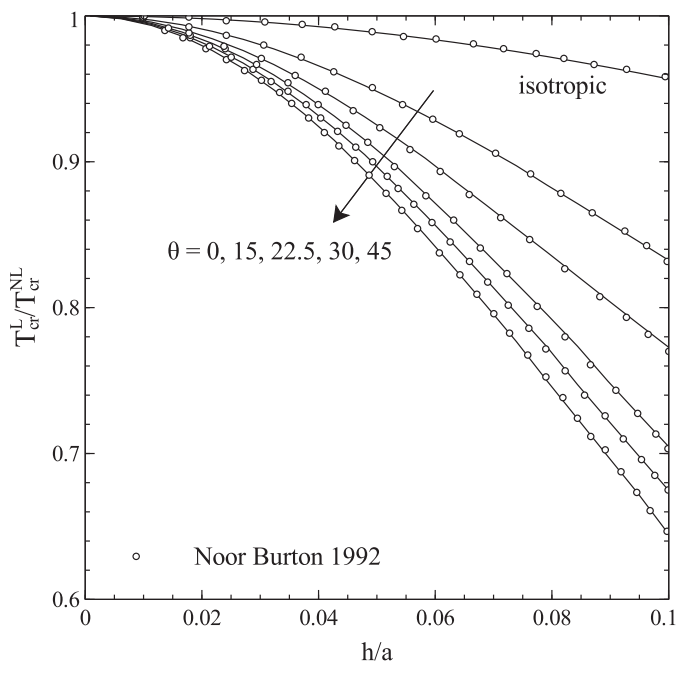

(a) of results are obtained with $20 \times 20$ Legendre functions. The results obtained using $\mathrm{ED}_{222}$ highlight, as already discussed, the superior performances of the Legendre polynomials. In particular, the critical temperature for $\theta$ equal to 45 is $3 \%$ smaller than the corresponding value obtained in Ref. [39] using trigonometric functions. Again, the difference reduces with the degree of anisotropy of the panel, and small deviations are seen for $\theta$ equal to 15 .

No significant improvements are achieved when $\mathrm{ED}_{554}$ and $\mathrm{LD}_{222}$ theories are considered, which is consistent with the relatively thin configuration under investigation. Similarly, the critical temperature obtained by accounting for the nonlinear prebuckling effects are very close to the linear ones, with a maximum difference of approximately $1 \%$. Note also that the linear prediction is always conservative, thus on the safe side.

Additional novel results are reported in Table 5 by considering the same material, lay-ups and boundary conditions of the previous example. The investigation is now extended to various length-to-width ratios. Nondimensional critical temperatures are reported for three distinct kinematic theories, with and without accounting for the effect of pre-buckling deformations. These results could prove to be useful as an additional benchmark for future investigations.

To further investigate the thermal buckling response of the panels analyzed in Table 4, the width-to-thickness ratio is plotted against the critical temperature ratio in Figs. 4-6. The effects due to ply angles, material orthotropy ratio and boundary conditions are addressed and, to the best of the authors' knowledge, no previous investigations have covered these aspects.

The plates here considered are made of material M2 and are obtained by the stacking of four plies with different angles of orientations. Orthotropic plates, where all the plies are oriented at

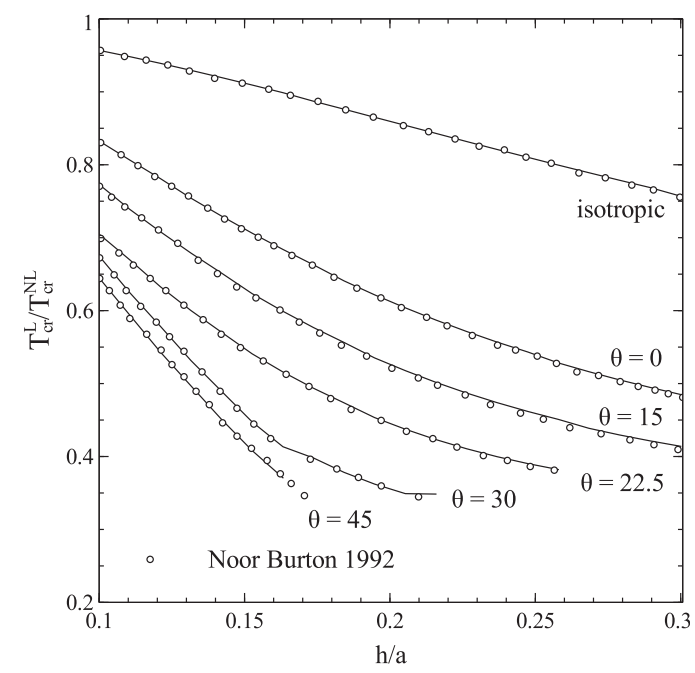

(b)

Fig. 3. Comparison of critical temperature ratios versus thickness-to-width ratio for plates with lay-up $[ \pm \theta]_{5}$ : (a) thin plates $(0 \leqslant h / a \leqslant 0.1)$, (b) thick plates $(0.1 \leqslant h / a \leqslant 0.3)$.

Table 4

Comparison of critical temperatures $T_{\text {cr }}(\mathrm{K})$ for square SSSS plates with $a / h=50$, material M2 and lay-up $[ \pm \theta]_{\mathrm{s}}$. Note: ${ }^{\text {ausing }} 12 \times 12$ trigonometric functions.

\begin{tabular}{|c|c|c|c|c|c|c|c|c|}
\hline \multirow[t]{2}{*}{$\theta$} & \multirow[t]{2}{*}{ Ref.[39] } & \multirow{2}{*}{$\begin{array}{l}\mathrm{ED}_{222}^{a} \\
\mathrm{~L}\end{array}$} & \multicolumn{2}{|c|}{$\mathrm{ED}_{222}$} & \multicolumn{2}{|c|}{$\mathrm{ED}_{554}$} & \multicolumn{2}{|c|}{$\mathrm{LD}_{222}$} \\
\hline & & & $\mathrm{L}$ & $\mathrm{NL}$ & $\mathrm{L}$ & $\mathrm{NL}$ & $\mathrm{L}$ & $\mathrm{NL}$ \\
\hline 0 & 118.128 & 118.128 & 118.129 & 119.150 & 117.906 & 118.923 & 117.907 & 118.924 \\
\hline 15 & 156.394 & 156.394 & 155.164 & 156.945 & 154.236 & 155.996 & 154.142 & 155.900 \\
\hline 30 & 245.693 & 245.693 & 240.702 & 245.018 & 238.145 & 242.369 & 237.803 & 242.015 \\
\hline 45 & 266.242 & 266.242 & 258.546 & 263.531 & 254.937 & 259.783 & 254.432 & 259.259 \\
\hline
\end{tabular}


Table 5

Critical temperatures $\alpha_{0} T_{\text {cr }}$ for square SSSS plates of material M2, lay-up $[ \pm \theta]_{\mathrm{S}}$.

\begin{tabular}{|c|c|c|c|c|c|c|c|}
\hline \multirow[t]{2}{*}{$a / h$} & \multirow[t]{2}{*}{$\theta$} & \multicolumn{2}{|c|}{$\mathrm{ED}_{222}$} & \multicolumn{2}{|c|}{$\mathrm{ED}_{554}$} & \multicolumn{2}{|c|}{$\mathrm{LD}_{222}$} \\
\hline & & $\mathrm{L}$ & NL & $\mathrm{L}$ & $\mathrm{NL}$ & $\mathrm{L}$ & $\mathrm{NL}$ \\
\hline \multirow[t]{5}{*}{10} & 0 & $6.1954^{-2}$ & $7.3979^{-2}$ & $5.9843^{-2}$ & $7.1025^{-2}$ & $5.9853^{-2}$ & $7.1038^{-2}$ \\
\hline & 15 & $7.8070^{-2}$ & $9.8009^{-2}$ & $7.2937^{-2}$ & $9.0149^{-2}$ & $7.2546^{-2}$ & $8.9560^{-2}$ \\
\hline & 22.5 & $9.8920^{-2}$ & $1.3321^{-1}$ & $8.9711^{-2}$ & $1.1697^{-1}$ & $8.8757^{-2}$ & $1.1539^{-1}$ \\
\hline & 30 & $1.2905^{-1}$ & $1.9305^{-1}$ & $1.1406^{-1}$ & $1.6198^{-1}$ & $1.1230^{-1}$ & $1.5856^{-1}$ \\
\hline & 45 & $1.3802^{-1}$ & $2.1579^{-1}$ & $1.2231^{-1}$ & $1.7965^{-1}$ & $1.2045^{-1}$ & $1.7576^{-1}$ \\
\hline \multirow[t]{5}{*}{20} & 0 & $1.8735^{-2}$ & $1.9726^{-2}$ & $1.8531^{-2}$ & $1.9500^{-2}$ & $1.8532^{-2}$ & $1.9501^{-2}$ \\
\hline & 15 & $2.4313^{-2}$ & $2.6004^{-2}$ & $2.3697^{-2}$ & $2.5302^{-2}$ & $2.3644^{-2}$ & $2.5241^{-2}$ \\
\hline & 22.5 & $3.1597^{-2}$ & $3.4502^{-2}$ & $3.0377^{-2}$ & $3.3055^{-2}$ & $3.0238^{-2}$ & $3.2891^{-2}$ \\
\hline & 30 & $3.8460^{-2}$ & $4.2858^{-2}$ & $3.6946^{-2}$ & $4.0990^{-2}$ & $3.6761^{-2}$ & $4.0764^{-2}$ \\
\hline & 45 & $4.1176^{-2}$ & $4.6303^{-2}$ & $3.9138^{-2}$ & $4.3697^{-2}$ & $3.8874^{-2}$ & $4.3370^{-2}$ \\
\hline \multirow[t]{5}{*}{50} & 0 & $3.1895^{-3}$ & $3.2171^{-3}$ & $3.1835^{-3}$ & $3.2109^{-3}$ & $3.1835^{-3}$ & $3.2109^{-3}$ \\
\hline & 15 & $4.1894^{-3}$ & $4.2375^{-3}$ & $4.1644^{-3}$ & $4.2119^{-3}$ & $4.1618^{-3}$ & $4.2093^{-3}$ \\
\hline & 22.5 & $5.4996^{-3}$ & $5.5828^{-3}$ & $5.4468^{-3}$ & $5.5284^{-3}$ & $5.4402^{-3}$ & $5.5215^{-3}$ \\
\hline & 30 & $6.4990^{-3}$ & $6.6155^{-3}$ & $6.4299^{-3}$ & $6.5440^{-3}$ & $6.4207^{-3}$ & $6.5344^{-3}$ \\
\hline & 45 & $6.9807^{-3}$ & $7.1153^{-3}$ & $6.8833^{-3}$ & $7.0141^{-3}$ & $6.8697^{-3}$ & $7.0000^{-3}$ \\
\hline \multirow[t]{5}{*}{100} & 0 & $8.0479^{-4}$ & $8.0655^{-4}$ & $8.0441^{-4}$ & $8.0616^{-4}$ & $8.0441^{-4}$ & $8.0617^{-4}$ \\
\hline & 15 & $1.0593^{-3}$ & $1.0623^{-3}$ & $1.0571^{-3}$ & $1.0601^{-3}$ & $1.0569^{-3}$ & $1.0599^{-3}$ \\
\hline & 22.5 & $1.3928^{-3}$ & $1.3980^{-3}$ & $1.3882^{-3}$ & $1.3934^{-3}$ & $1.3875^{-3}$ & $1.3928^{-3}$ \\
\hline & 30 & $1.6382^{-3}$ & $1.6455^{-3}$ & $1.6318^{-3}$ & $1.6390^{-3}$ & $1.6309^{-3}$ & $1.6381^{-3}$ \\
\hline & 45 & $1.7607^{-3}$ & $1.7691^{-3}$ & $1.7515^{-3}$ & $1.7598^{-3}$ & $1.7502^{-3}$ & $1.7585^{-3}$ \\
\hline
\end{tabular}

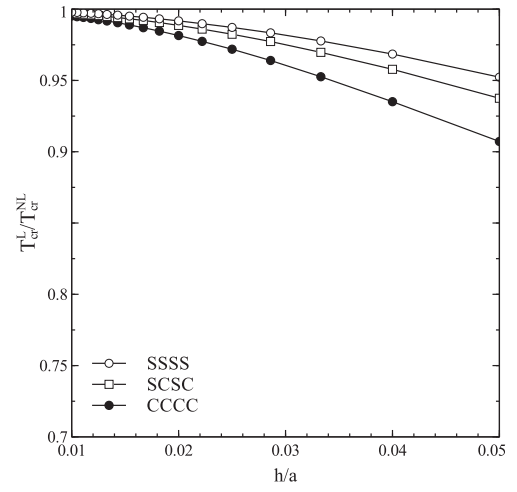

(a)

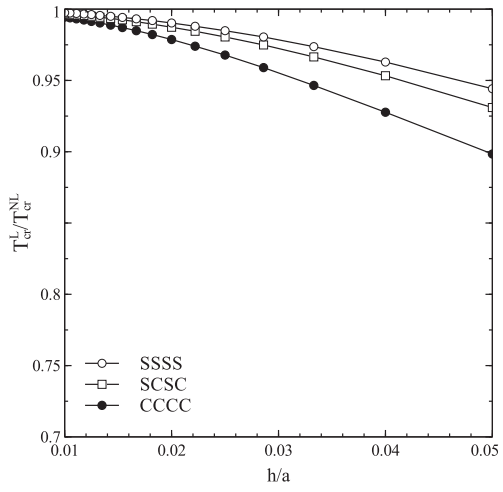

(b)

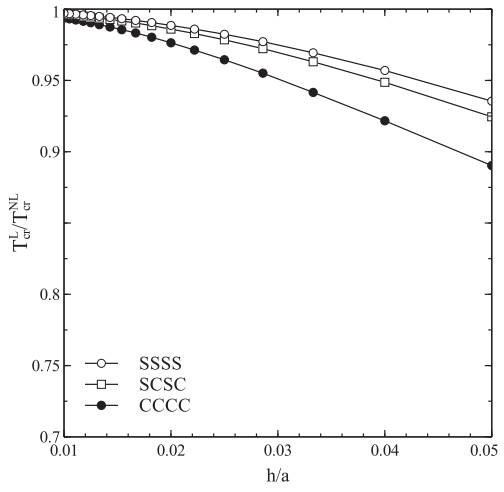

(c)

Fig. 4. Critical temperature ratio versus thickness-to-width ratio for plates with lay-up $[0]_{4}$ : (a) $E_{11} / E_{22}=10$, (b) $E_{11} / E_{22}=15$, (c) $E_{11} / E_{22}=20$.

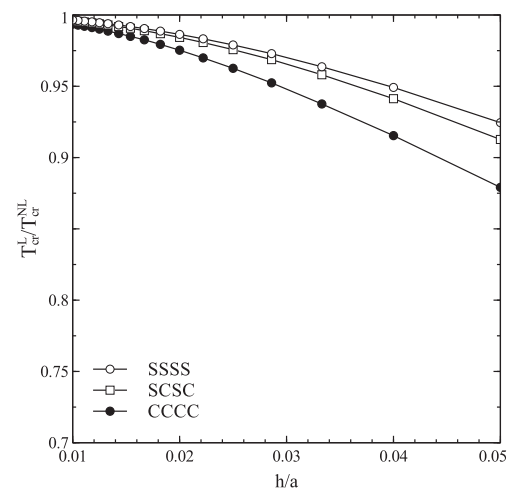

(a)

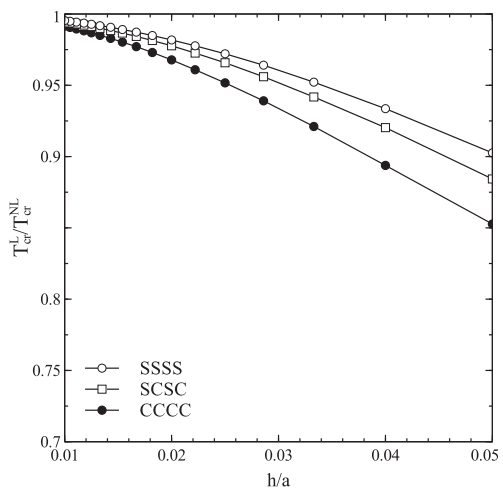

(b)

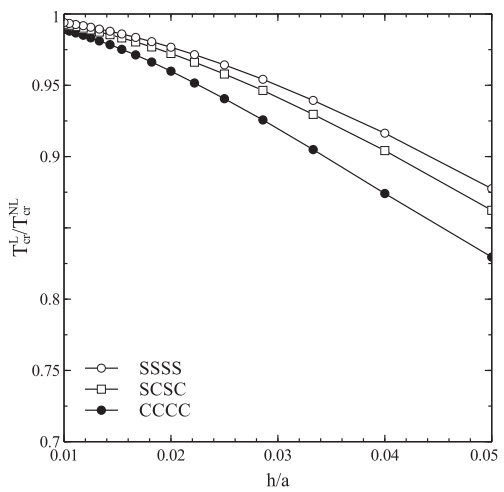

(c)

Fig. 5. Critical temperature ratio versus thickness-to-width ratio for plates with lay-up $[ \pm 22.5]_{s}$, Material M2: (a) $E_{11} / E_{22}=10$, (b) $E_{11} / E_{22}=15$, (c) $E_{11} / E_{22}=20$.

0 , are considered in Fig. 4, while angle-ply configurations with ply angles at 22.5 and 45 are assumed for the plots of Figs. 5 and 6. As done in the previous analyses, the computations are performed by considering 20 functions along both the directions, while theory
$\mathrm{ED}_{554}$ is assumed. In agreement with the results of Ref. [28], a reduction of the critical temperature ratio is observed for increasing values of $h / a$ and $\theta$. In addition, the effect of the boundary condition can be clearly noticed from the plots, where SSSS, SCSC and 


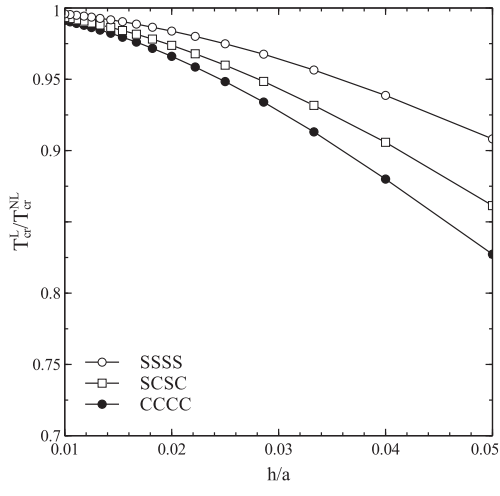

(a)

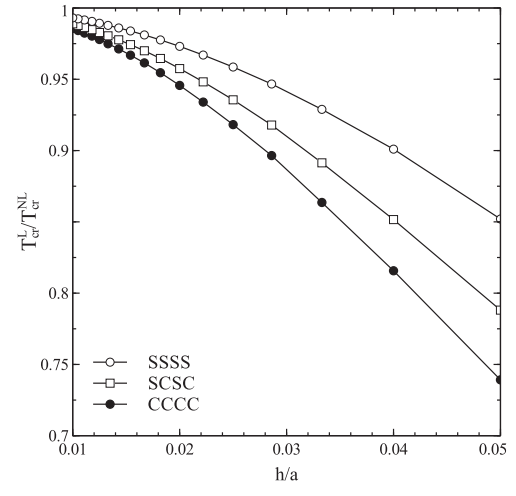

(b)

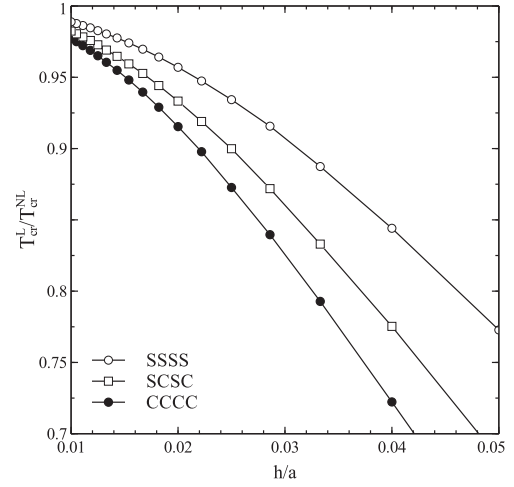

(c)

Fig. 6. Critical temperature ratio versus thickness-to-width ratio for plates with lay-up $[ \pm 45]_{s}$, Material M2: (a) $E_{11} / E_{22}=10$, (b) $E_{11} / E_{22}=15$, (c) $E_{11} / E_{22}=20$.

Table 6

Comparison of critical temperatures $\alpha_{0} T_{\text {cr }}$ for skew plates made of material M1, $a / b=1$ and $a / h=10$.

\begin{tabular}{|c|c|c|c|c|c|c|c|c|c|}
\hline \multirow[b]{2}{*}{ Lay-up } & \multirow[b]{2}{*}{$\Psi$} & \multicolumn{4}{|c|}{ CSCS } & \multicolumn{4}{|c|}{$\mathrm{CCCC}$} \\
\hline & & Ref. [22] & $\mathrm{ED}_{332}$ & $\mathrm{ED}_{554}$ & $\mathrm{LD}_{222}$ & Ref. [22] & $\mathrm{ED}_{332}$ & $\mathrm{ED}_{554}$ & $\mathrm{LD}_{222}$ \\
\hline \multirow[t]{4}{*}[0/90]{$_{s}$} & 0 & 0.1305 & 0.1317 & 0.1306 & 0.1302 & 0.1601 & 0.1616 & 0.1603 & 0.1597 \\
\hline & 15 & 0.1340 & 0.1354 & 0.1341 & 0.1337 & 0.1618 & 0.1634 & 0.1620 & 0.1615 \\
\hline & 30 & 0.1447 & 0.1456 & 0.1451 & 0.1446 & 0.1690 & 0.1710 & 0.1693 & 0.1687 \\
\hline & 45 & 0.1523 & 0.1538 & 0.1526 & 0.1521 & 0.1893 & 0.1926 & 0.1896 & 0.1889 \\
\hline \multirow[t]{4}{*}[\pm45]{$_{s}$} & 0 & 0.1360 & 0.1364 & 0.1321 & 0.1309 & 0.1609 & 0.1623 & 0.1573 & 0.1561 \\
\hline & 15 & 0.1427 & 0.1433 & 0.1386 & 0.1372 & 0.1678 & 0.1693 & 0.1639 & 0.1625 \\
\hline & 30 & 0.1625 & 0.1635 & 0.1576 & 0.1561 & 0.1886 & 0.1905 & 0.1838 & 0.1822 \\
\hline & 45 & 0.1950 & 0.1972 & 0.1893 & 0.1875 & 0.2249 & 0.2275 & 0.2185 & 0.2165 \\
\hline \multirow[t]{4}{*}[\pm45]{$_{2 s}$} & 0 & 0.1534 & 0.1539 & 0.1535 & 0.1498 & 0.1809 & 0.1819 & 0.1816 & 0.1779 \\
\hline & 15 & 0.1611 & 0.1616 & 0.1611 & 0.1571 & 0.1887 & 0.1897 & 0.1894 & 0.1854 \\
\hline & 30 & 0.1835 & 0.1842 & 0.1835 & 0.1786 & 0.2121 & 0.2133 & 0.2130 & 0.2080 \\
\hline & 45 & 0.2199 & 0.2208 & 0.2199 & 0.2134 & 0.2526 & 0.2543 & 0.2537 & 0.2468 \\
\hline
\end{tabular}

CCCC conditions are considered. The critical temperature ratio is seen to decrease when constraints are added to the plate. So, the simply-supported condition represents the upper bound case, while the fully clamped is the lower bound. Also it can be argued from Figs. 4-6 that highest amounts of the material orthotropy ratios have the effect of reducing the critical temperature ratio.

\subsubsection{Example 3}

To illustrate the generality of the proposed implementation, the results are now presented for a set of skew composite plates subjected to arbitrary boundary conditions. The panels are characterized by the geometric ratios $a / b=1, a / h=10$ and skew angle $\Psi$; the thermoelastic properties are those of material M1. The comparison is presented between the results obtained with different theories and the critical temperatures obtained by Kant and Babu [22] using HSDT finite elements. The results are summarized in Table 6 for two different sets of boundary condition, CSCS and CCCC. The first and the third entries refer to the constraints of the oblique sides of the panels, which are clamped in both cases. Very close agreement can be noticed between the present results and those of Ref. [22].

\subsection{Sandwich plates}

\subsubsection{Example 4}

Results are now presented for sandwich configurations. The first assessment regards the cross-ply configuration discussed in Refs. [30,39]. It consists of a square sandwich panel with tenlayer face-sheets made of material M3 with stacking sequence
$[0 / 90]_{5 s}$. The 0 plies are at the outer top and bottom surface, guaranteeing the symmetry of the panel stacking sequence. The core properties are those of material $\mathrm{C} 1$. The panel is simplysupported along its four edges. Two nondimensional parameters are made vary in the analyses, namely the length-to-thickness ratio $a / h$, and the ratio between the face-sheet thickness versus the panel thickness $h_{\mathrm{f}} / h$.

It is well known that the need for refined theories becomes increasingly relevant as the values of $a / h$ diminish and those of $h_{\mathrm{f}} / h$ increase. In fact, a refined transverse response is more important when the plate becomes thick and the stress gradients become more abrupt, which occurs when the stiff faces increase their thickness in comparison with the soft core. The results are summarized in Table 7, and are obtained by considering different theories and different shape functions. In particular, the first set of results is obtained by considering the layerwise $\mathrm{LD}_{222}$ theory in conjunction with a trigonometric expansion. Calculations are performed by forcing the buckling mode to be of global type, i.e. assuming a deflected pattern characterized by one single half-wave along the two orthogonal directions $x$ and $y$. Under this assumption, local instabilities are prevented and close matching with the reference results is achieved. This combination of kinematic theory and shape functions is the same proposed by Fazzolari and Carrera in [39] and, in fact, perfect matching is obtained with the results obtained by the authors. Good agreement is also observed with the 3D results of Ref. [30]. Again, it is worth highlighting that the exact solution of this problem is characterized by a trigonometric displacement field, thus one single trigonometric function can capture the exact shape of one of the buckling modes. The remaining 
Table 7

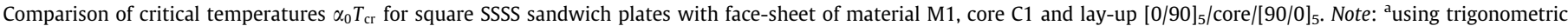
functions, *local modes.

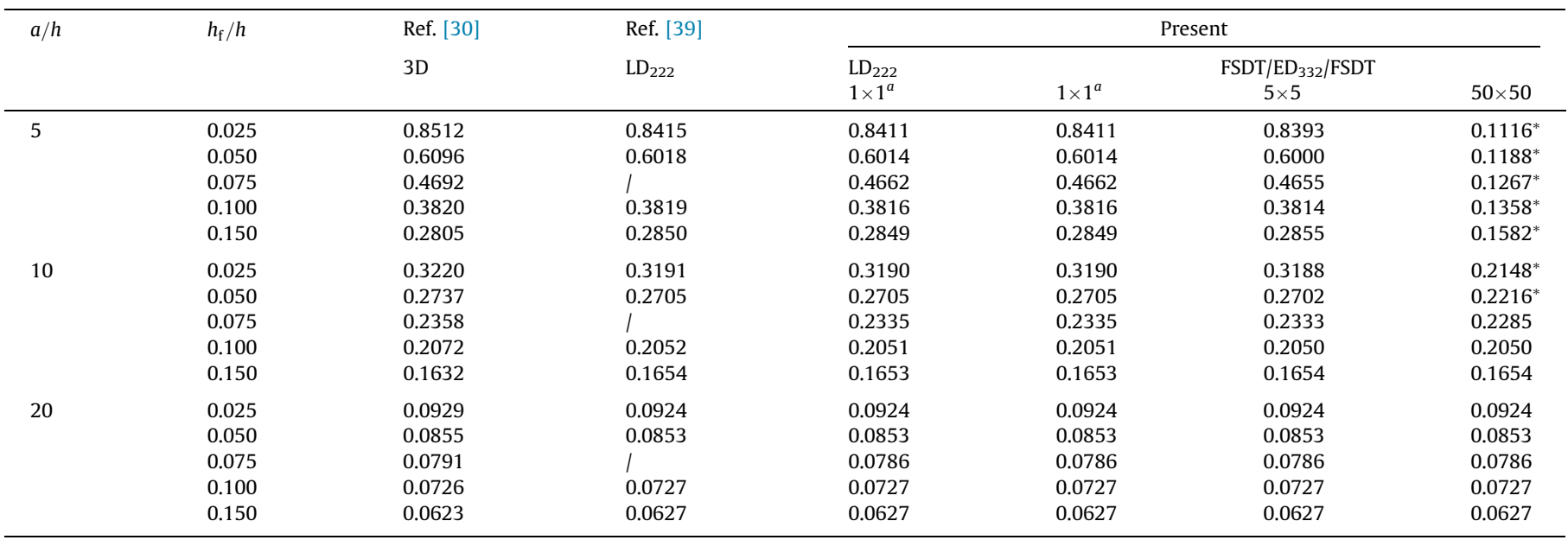

results in the table are calculated by adopting a sublaminate description of the panel, where the face-sheets are modeled with FSDT theory, and the core with $\mathrm{ED}_{332}$. In this case, the number of theory-related degrees of freedom is equal to 15, against the 189 required by $\mathrm{LD}_{222}$. As seen form Table 7 , the accuracy of the predictions is not affected by the drastic reduction of the problem size, and the results are identical to those computed with $\mathrm{LD}_{222}$. The advantage of a sublaminate representation against a purely layerwise is thus clear. In the last two columns, Legendre-type functions are adopted, with 5 and 50 terms along the two directions. The results obtained with 5 functions are in good agreement with those obtained with 1 trigonometric function. The predicted buckling modes are still of global type, and the corresponding eigenvalues resemble those previously obtained. However, local buckling modes are detected - they are labeled with an asterisk in Table 7 - when the number of functions is raised up to 50. These modes are observed for the thickest configurations and were not reported in past studies due, presumably, to the premature truncation of the series to approximate the unknown fields. The results here reported are those obtained using 50 functions, but the authors cannot exclude that lower eigenvalues can be found if the expansion is further enriched. Given the size of the problem and the computational costs associated with a larger number of functions, ad hoc analysis tools would be needed.

\subsubsection{Example 5}

The critical temperatures of angle-ply sandwich configurations are presented in Table 8, where the comparison is presented against the results obtained by Matsunaga [26]. The face-sheets have lay-up $[ \pm \theta]_{5}$, and are realized with material M3, with the plies oriented at $+\theta$ on the external faces. The core is made of material C1. Simply-supported boundary conditions are assumed. The results are obtained by considering a purely layerwise approach $\mathrm{LD}_{222}$, and a sublaminate description FSDT/ED ${ }_{332} / \mathrm{FSDT}$. For consistency with Ref. [26], a first set of results is derived by considering $2 \times 1$ trigonometric functions. The two remaining set of analyses are performed with Legendre polynomials and $5 \times 5$ and $50 \times 50$ functions.

The results demonstrate relatively good agreement with Matsunaga, as the present predictions are slightly smaller. The reason for this discrepancy is believed to be related with the higher refinement of the theories here considered, both in the case of the layerwise approach and the sublaminate one, in contrast to the equivalent single layer description of Ref. [26]. Also for this case, the results demonstrate the excellent accuracy versus computational cost of the sublaminate models. As a matter of fact, the critical temperatures are as accurate as those determined with a layerwise approach, while requiring less than one tenth of the theory-related degrees of freedom. As seen from Table 8, local

Table 8

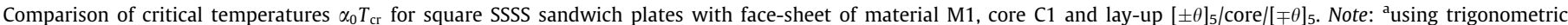
functions, *local modes.

\begin{tabular}{|c|c|c|c|c|c|c|c|}
\hline \multirow[t]{2}{*}{$a / h$} & \multirow[t]{2}{*}{$h_{\mathrm{f}} / h$} & \multirow[t]{2}{*}{$\theta$} & \multirow[t]{2}{*}{ Ref. [26] } & \multirow{2}{*}{$\begin{array}{l}\mathrm{LD}_{222} \\
2 \times 1^{a}\end{array}$} & \multicolumn{3}{|c|}{$\mathrm{FSDT} / \mathrm{ED}_{332} / \mathrm{FSDT}$} \\
\hline & & & & & $2 \times 1^{a}$ & $5 \times 5$ & $50 \times 50$ \\
\hline \multirow[t]{4}{*}{5} & \multirow[t]{4}{*}{0.15} & 0 & 0.1837 & 0.1757 & 0.1739 & 0.1741 & $0.1582^{*}$ \\
\hline & & 15 & 0.2109 & 0.2037 & 0.2024 & 0.2023 & $0.1582^{*}$ \\
\hline & & 30 & 0.2702 & 0.2610 & 0.2609 & 0.2608 & $0.1582^{*}$ \\
\hline & & 45 & 0.3311 & 0.3216 & 0.3228 & 0.3225 & $0.1581^{*}$ \\
\hline \multirow[t]{4}{*}{10} & \multirow[t]{4}{*}{0.15} & 0 & 0.09938 & 0.0960 & 0.0955 & 0.0957 & 0.0955 \\
\hline & & 15 & 0.1285 & 0.1258 & 0.1254 & 0.1254 & 0.1252 \\
\hline & & 30 & 0.1916 & 0.1874 & 0.1873 & 0.1873 & 0.1871 \\
\hline & & 45 & 0.2201 & 0.2174 & 0.2178 & 0.2176 & 0.2175 \\
\hline \multirow[t]{4}{*}{20} & \multirow[t]{4}{*}{0.15} & 0 & 0.03820 & 0.0373 & 0.0373 & 0.0374 & 0.0373 \\
\hline & & 15 & 0.05530 & 0.0551 & 0.0551 & 0.0551 & 0.0550 \\
\hline & & 30 & 0.08845 & 0.0893 & 0.0894 & 0.0894 & 0.0893 \\
\hline & & 45 & 0.09679 & 0.0977 & 0.0978 & 0.0978 & 0.0977 \\
\hline
\end{tabular}




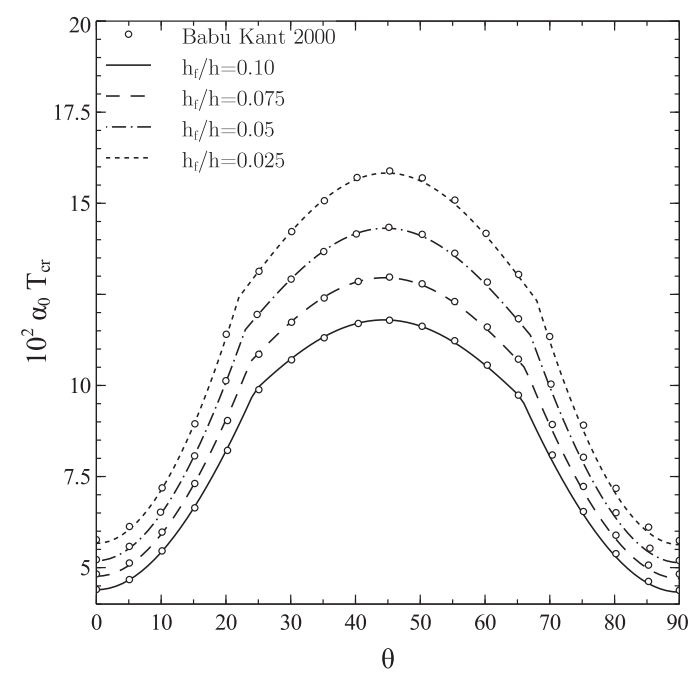

(a)

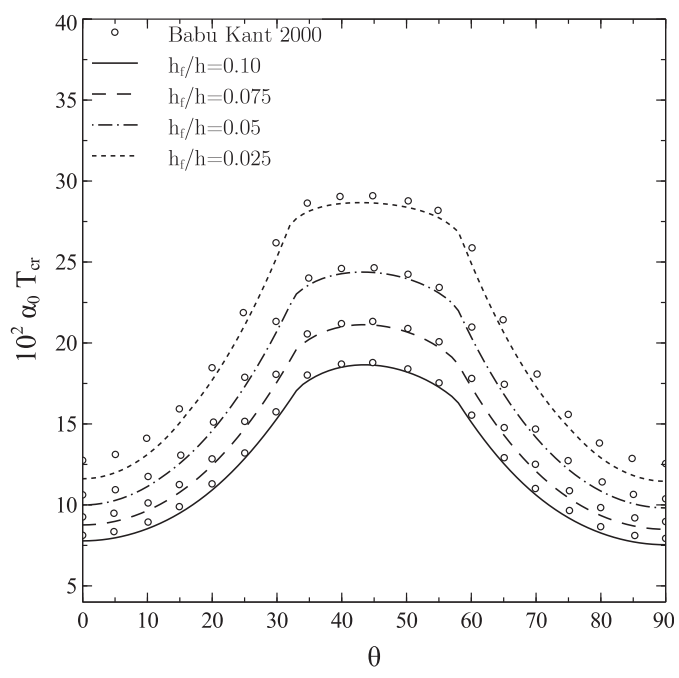

(b)

Fig. 7. Comparison of critical temperatures for angle-ply sandwich plates with $a / h=20$, lay-up $[ \pm \theta]_{5} /$ core $/[\mp \theta]_{5}$ and different boundary conditions: (a) SSSS, (b) CCCC.

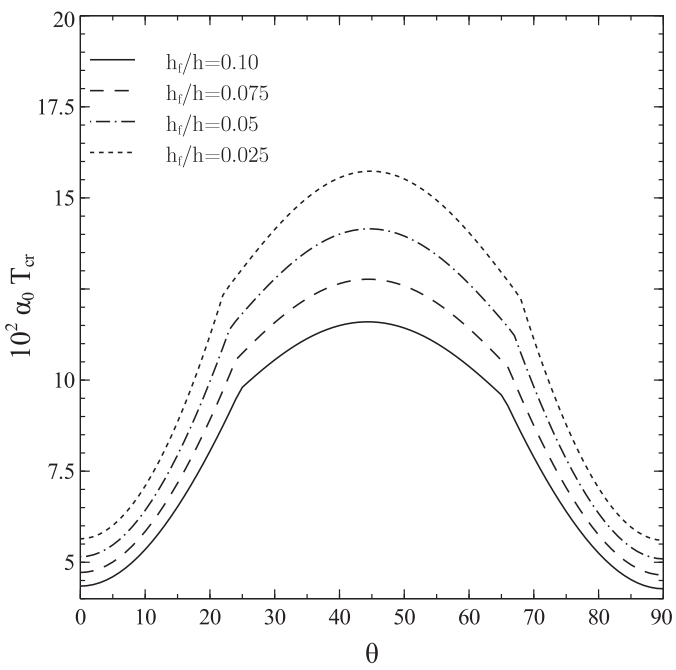

(a)

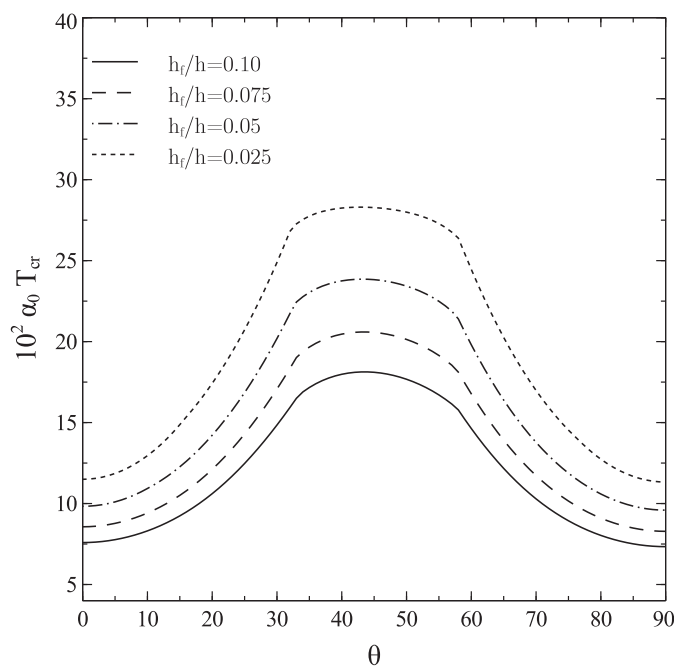

(b)

Fig. 8. Critical temperatures obtained with $\mathrm{ED}_{332} / \mathrm{ED}_{332} / \mathrm{ED}_{332}$ for sandwich plates with $a / h=20$, lay-up $[ \pm \theta]_{5} /$ core $/[\mp \theta]_{5}$ and different boundary conditions: (a) $\operatorname{SSSS}$, (b) CCCC.

buckling modes are detected when increasing the number of functions up to 50 for two of the configurations analyzed, i.e. a thick plate $(\mathrm{a} / \mathrm{h}=5)$ and $\theta$ equal to 30 .

\subsubsection{Example 6}

Further results are proposed in Fig. 7, where the nondimensional critical temperatures are reported for sandwich plates with same materials and lay-ups as in the previous example, and a geometric ratio $a / h$ fixed at 20 . The results for simply-supported conditions are presented in Fig. 7(a), while those of a fully clamped panel in Fig. 7(b). The comparison is reported against the results obtained by Babu and Kant [21] that utilized HOST9 isoparametric finite elements, based on third-order description of the in-plane displacement components, and a zero order representation of the out-of-plane displacement component. For consistency, the results are firstly computed with $\mathrm{ED}_{330}$ theory, and a number of $20 \times 20$ functions. The results reveal good correspondence with Babu and Kant, and the effects of the ply orientation and the face- to-thickness ratio on the panel critical temperature are correctly captured. Also the effect of different boundary conditions is in agreement with Ref. [21]. With the aim of providing additional results to be used for future benchmarking, the plots obtained using a sublaminate theory $\mathrm{ED}_{332} / \mathrm{ED}_{332} / \mathrm{ED}_{332}$ are presented in Fig. 8. In addition, the results are summarized in tabular form in Table 9.

\subsubsection{Example 7}

Finally, an investigation over the effect of the pre-buckling deformations is discussed with regard to sandwich plates. In particular, the critical temperature ratio is calculated for angle-ply configurations with face-sheets of material M3 at $[ \pm \theta]_{5}$ and core of material $\mathrm{C} 1$. The results are obtained by means of a sublaminate $\mathrm{ED}_{332} / \mathrm{ED}_{332} / \mathrm{ED}_{332}$ description of the panel, chosen to guarantee high accuracy of the results.

The charts relative to a ply angle $\theta$ of 22.5 are reported in Fig. 9, where two distinct values of $h_{\mathrm{f}} / h$ are assumed. In particular, they 
Table 9

Nondimensional critical temperatures $10^{2} \alpha_{0} T_{\mathrm{cr}}$ obtained with $\mathrm{ED}_{332} / \mathrm{ED}_{332} / \mathrm{ED}_{332}$ for sandwich plates with $a / h=20$, lay-up $[ \pm \theta]_{5} / \mathrm{core} /[\mp \theta]_{5}$.

\begin{tabular}{|c|c|c|c|c|c|}
\hline & $\theta$ & $\begin{array}{l}h_{\mathrm{f}} / h \\
0.10\end{array}$ & 0.075 & 0.05 & 0.025 \\
\hline \multirow[t]{7}{*}{ SSSS } & 0 & 4.3453 & 4.7196 & 5.1514 & 5.6427 \\
\hline & 15 & 6.5230 & 7.1710 & 7.9427 & 8.8509 \\
\hline & 30 & 10.5539 & 11.5789 & 12.7781 & 14.1329 \\
\hline & 45 & 11.5973 & 12.7675 & 14.1507 & 15.7346 \\
\hline & 60 & 10.3761 & 11.4151 & 12.6426 & 14.0480 \\
\hline & 75 & 6.3944 & 7.0472 & 7.8342 & 8.7758 \\
\hline & 90 & 4.2727 & 4.6508 & 5.0922 & 5.6024 \\
\hline \multirow[t]{7}{*}{$\mathrm{CCCC}$} & 0 & 7.5896 & 8.5643 & 9.8306 & 11.4972 \\
\hline & 15 & 9.2393 & 10.5055 & 12.2680 & 14.8725 \\
\hline & 30 & 14.8500 & 17.0741 & 20.1931 & 24.8912 \\
\hline & 45 & 18.1054 & 20.5678 & 23.8258 & 28.2761 \\
\hline & 60 & 14.6330 & 16.7751 & 19.8024 & 24.4604 \\
\hline & 75 & 8.9355 & 10.1530 & 11.8755 & 14.5345 \\
\hline & 90 & 7.3373 & 8.2830 & 9.5915 & 11.3277 \\
\hline
\end{tabular}

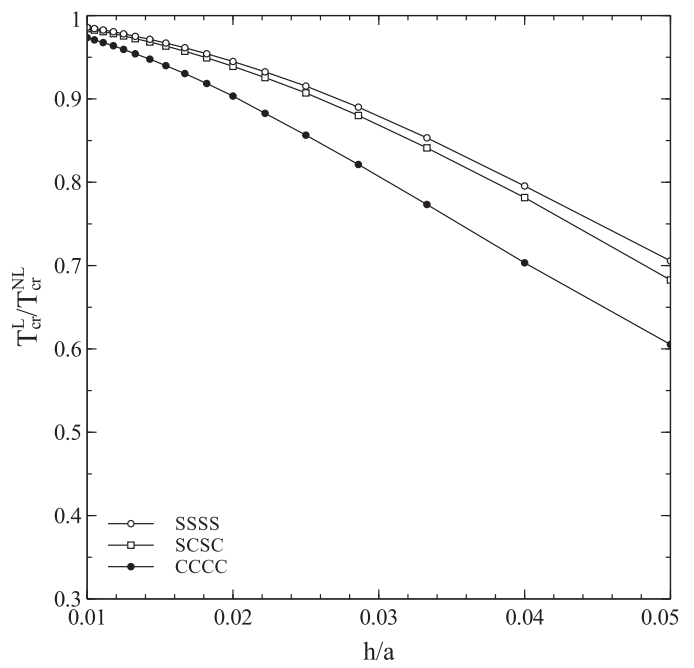

(a)

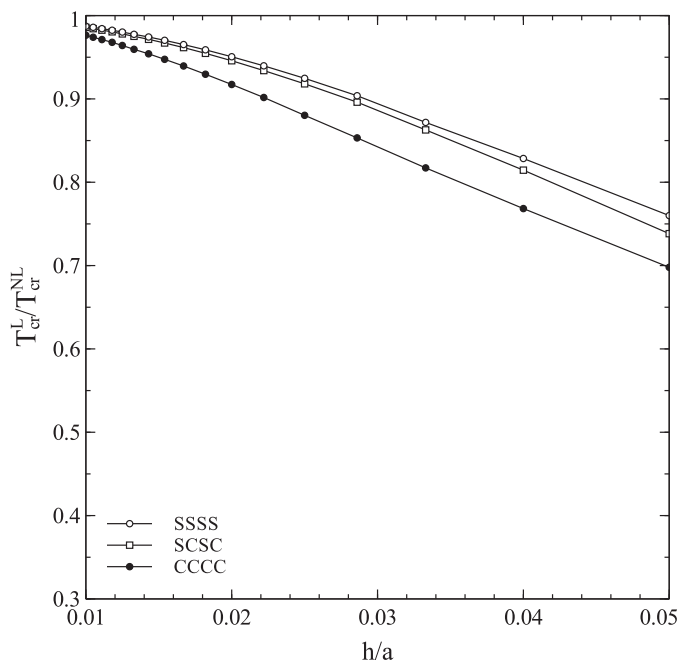

(b)

Fig. 9. Critical temperature ratios for sandwich plates with $[ \pm 22.5]_{5} /$ core $/[\mp 22.5]_{5}$, and different face-sheet ratios: $h_{\mathrm{f}} / h=0.05$, $(\mathrm{b}) h_{\mathrm{f}} / h=0.10$.

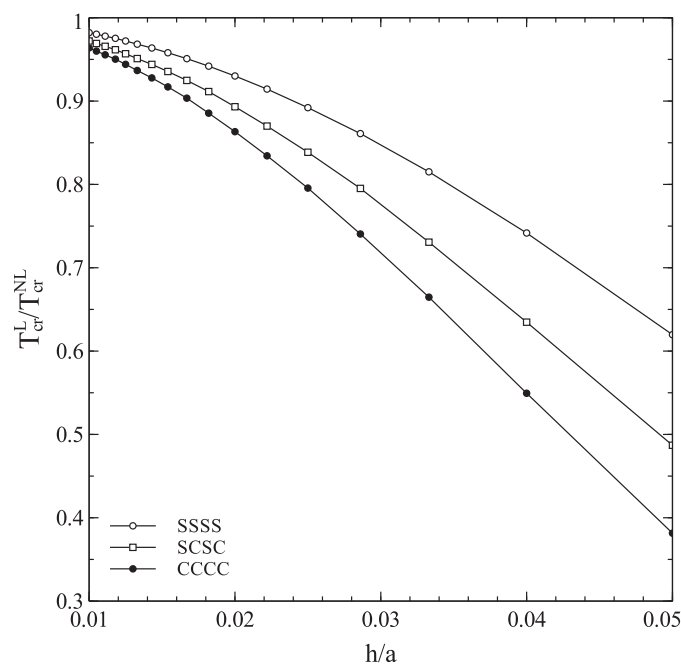

(a)

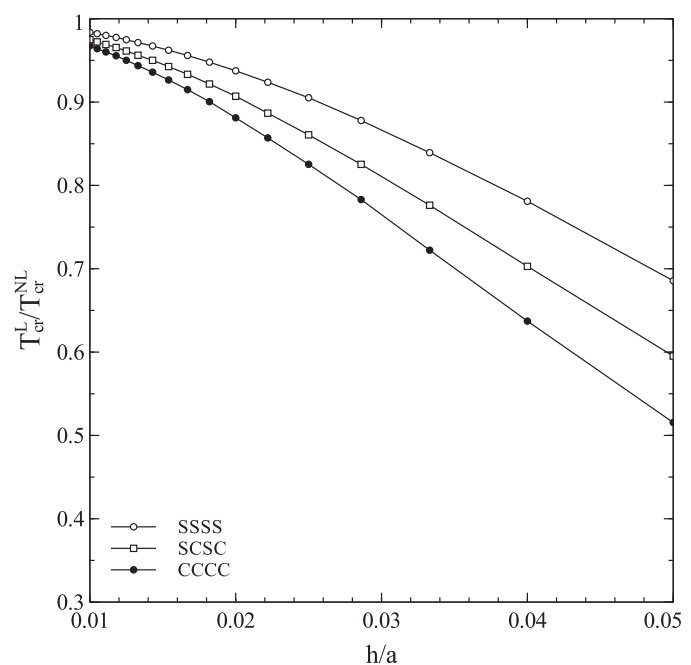

(b)

Fig. 10. Critical temperature ratios for sandwich plates with $[ \pm 45]_{5} /$ core $/[\mp 45]_{5}$, and different face-sheet ratios: $h_{\mathrm{f}} / h=0.05,(\mathrm{~b}) h_{\mathrm{f}} / h=0.10$. 
Table 10

Critical temperature ratios $T_{\mathrm{cr}}^{\mathrm{L}} / T_{\mathrm{cr}}^{\mathrm{NL}}$ for sandwich plates with $[ \pm \theta]_{5} /$ core $/[\mp \theta]_{5}$.

\begin{tabular}{|c|c|c|c|c|c|c|c|}
\hline \multirow[t]{2}{*}{$\theta$} & \multirow[t]{2}{*}{$h / a$} & \multicolumn{3}{|c|}{$\underline{h_{\mathrm{f}} / h=0.05}$} & \multicolumn{3}{|c|}{$\underline{h_{\mathrm{f}} / h=0.10}$} \\
\hline & & SSSS & SCSC & $\mathrm{CCCC}$ & SSSS & SCSC & $\mathrm{CCCC}$ \\
\hline \multirow[t]{5}{*}{22.5} & $1 / 20$ & 0.7057 & 0.6827 & 0.6053 & 0.7600 & 0.7383 & 0.6979 \\
\hline & $1 / 40$ & 0.9154 & 0.9072 & 0.8565 & 0.9247 & 0.9181 & 0.8803 \\
\hline & $1 / 60$ & 0.9614 & 0.9572 & 0.9304 & 0.9652 & 0.9616 & 0.9395 \\
\hline & $1 / 80$ & 0.9781 & 0.9756 & 0.9594 & 0.9802 & 0.9780 & 0.9641 \\
\hline & $1 / 100$ & 0.9859 & 0.9843 & 0.9735 & 0.9872 & 0.9858 & 0.9764 \\
\hline \multirow[t]{5}{*}{45} & $1 / 20$ & 0.6197 & 0.4870 & 0.3815 & 0.6856 & 0.5952 & 0.5155 \\
\hline & $1 / 40$ & 0.8921 & 0.8386 & 0.7956 & 0.9052 & 0.8607 & 0.8253 \\
\hline & $1 / 60$ & 0.9509 & 0.9248 & 0.9035 & 0.9559 & 0.9333 & 0.9149 \\
\hline & $1 / 80$ & 0.9721 & 0.9568 & 0.9441 & 0.9748 & 0.9613 & 0.9501 \\
\hline & $1 / 100$ & 0.9821 & 0.9721 & 0.9638 & 0.9838 & 0.9749 & 0.9675 \\
\hline
\end{tabular}

are taken equal to 0.05 in Fig. 9(a) and 0.10 in Fig. 9(b). Similarly, the case of $\theta$ equal to 45 is considered in Fig. 10.

The results demonstrate the same trends observed for the monolithic plates. However, the effect of pre-buckling deformations is much more relevant in the case of sandwich panels. The critical temperature ratio diminishes for increasing values of $\theta$ and $h / a$, thus thick configurations are those associated with more relevant pre-buckling effects. The same role is played by the introduction of constraints, and fully clamped panels are those characterized by the lowest temperature ratios. As seen from the four charts on Figs. 9 and 10, higher ratios $h_{\mathrm{f}} / h$ have the effect of reducing the relevance of the pre-buckling effects. For facilitating future benchmarking, the results of Figs. 9 and 10 are summarized in Table 10

\section{Conclusions}

A variable-kinematics model based on the Sublaminate Generalized Unified Formulation has been presented with regard to the thermal buckling analysis. The solution procedure is based on the Ritz method, where both Legendre polynomials and trigonometric shape functions were considered for approximating the displacement field. The results illustrate the advantages offered by Legendre polynomials in terms of rapidity of convergence. Exceptions are clearly represented by those case for which the exact solution is trigonometric.

Main advantage of the proposed approach consists in the flexibility offered to the analyst to select different kinematic theories for different clusters of plies. This possibility can be exploited for optimizing the accuracy versus the number of theory-related degrees of freedom, with consequent beneficial effects on the time for the analysis. The accuracy of the results has been demonstrated by comparison against reference solutions, including threedimensional results, both for monolithic and sandwich plates. In this second case, a proper choice of the sublaminate theories allowed to derive critical temperatures with accuracy comparable to the layerwise predictions, but demanding for much less degrees of freedom.

The excellent accuracy of the results is not restricted to the linear eigenvalue predictions, but includes the nonlinear results obtained by considering the pre-buckling deformations. With this regard, and in light of the few results available in the literature, novel reference solutions were presented with and without accounting for the pre-buckling effects. The role played by the lay-up anisotropy, boundary conditions, orthotropy and lengthto-thickness ratios was illustrated. The results here obtained do not account for temperature-dependent properties, which may have a relevant impact on the cases here considered, and which is subject of future investigations.

\section{Appendix A. Appendix}

\section{A.1. Through-the-thickness integrals}

Denote the assembly procedure of the thickness integrals with the following notation:

$\langle\cdot\rangle=\mathrm{A}_{k}^{N^{k}} \mathrm{~A}_{p}^{N_{p}^{k}}$.

where the assembly is performed twice, at ply and at sublaminate level. The resulting expressions of the thicknesswise contributions are:

$$
\begin{aligned}
& \mathbf{Z}_{u_{x} \partial u_{z} 13}^{0}=\left\langle\alpha^{p, k} \widetilde{C}_{13}^{p, k} \mathbf{Z}_{u_{x} \partial u_{z}}^{p, k}\right\rangle \quad \mathbf{Z}_{u_{x} \partial u_{z} 36}^{0}=\left\langle\alpha^{p, k} \widetilde{C}_{36}^{p, k} \mathbf{Z}_{u_{x} \partial u_{z}}^{p, k}\right\rangle \\
& \mathbf{Z}_{\partial u_{x} u_{z} 45}^{0}=\left\langle\alpha^{p, k} \widetilde{C}_{45}^{p, k} \mathbf{Z}_{\partial u_{x} u_{z}}^{p, k}\right\rangle \quad \mathbf{Z}_{\partial u_{x} u_{z} 55}^{0}=\left\langle\alpha^{p, k} \widetilde{C}_{55}^{p, k} \mathbf{Z}_{\partial u_{x} u_{z}}^{p, k}\right\rangle \\
& \mathbf{Z}_{\partial u_{y} u_{z} 44}^{0}=\left\langle\alpha^{p, k} \widetilde{C}_{44}^{p, k} \mathbf{Z}_{\partial u_{y} u_{z}}^{p, k}\right\rangle \quad \mathbf{Z}_{\partial u_{y} u_{z} 45}^{0}=\left\langle\alpha^{p, k} \widetilde{C}_{45}^{p, k} \mathbf{Z}_{\partial u_{y} u_{z}}^{p, k}\right\rangle \\
& \mathbf{Z}_{u_{y} \partial u_{z} 23}^{0}=\left\langle\alpha^{p, k} \widetilde{C}_{23}^{p, k} \mathbf{Z}_{u_{y} \partial u_{z}}^{p, k}\right\rangle \quad \mathbf{Z}_{u_{y} \partial u_{z} 36}^{0}=\left\langle\alpha^{p, k} \widetilde{C}_{36}^{p, k} \mathbf{Z}_{u_{y} \partial u_{z}}^{p, k}\right\rangle \\
& \mathbf{Z}_{u_{z} u_{z} 44}^{0}=\left\langle\beta^{p, k} \widetilde{C}_{44}^{p, k} \mathbf{Z}_{u_{z} u_{z}}^{p, k}\right\rangle \quad \mathbf{Z}_{u_{z} u_{z} 45}^{0}=\left\langle\beta^{p, k} \widetilde{C}_{45}^{p, k} \mathbf{Z}_{u_{z} u_{z}}^{p, k}\right\rangle \\
& \mathbf{Z}_{u_{z} u_{z} 55}^{0}=\left\langle\beta^{p, k} \widetilde{C}_{55}^{p, k} \mathbf{Z}_{u_{z} u_{z}}^{p, k}\right\rangle \quad \mathbf{Z}_{\partial u_{z} \partial u_{z} 33}^{0}=\left\langle\beta^{p, k} \widetilde{C}_{33}^{p, k} \mathbf{Z}_{\partial u_{z} \partial u_{z}}^{p, k}\right\rangle
\end{aligned}
$$

where:

$$
\begin{array}{lll}
\alpha^{p, k}={ }_{0} u_{z, z}^{p, k} & \beta^{p, k}=2{ }_{0} u_{z, z}^{p, k}+\left({ }_{0} u_{z, z}^{p, k}\right)^{2} \\
\mathbf{Z}_{u_{x} u_{x} 11}^{G}=\left\langle{ }_{0} \sigma_{x x}^{p, k} \mathbf{Z}_{u_{x} u_{x}}^{p, k}\right\rangle & \mathbf{Z}_{u_{x} u_{x} 22}^{G}=\left\langle{ }_{0} \sigma_{y y}^{p, k} \mathbf{Z}_{u_{x} u_{x}}^{p, k}\right\rangle & \mathbf{Z}_{u_{x} u_{x} 12}^{G}=\left\langle{ }_{0} \sigma_{x y}^{p, k} \mathbf{Z}_{u_{x} u_{x}}^{p, k}\right\rangle \\
\mathbf{Z}_{u_{y} u_{y} 11}^{G}=\left\langle{ }_{0} \sigma_{x x}^{p, k} \mathbf{Z}_{u_{y} u_{y}}^{p, k}\right\rangle & \mathbf{Z}_{u_{y} u_{y} 22}^{G}=\left\langle{ }_{0} \sigma_{y y}^{p, k} \mathbf{Z}_{u_{y} u_{y}}^{p, k}\right\rangle & \mathbf{Z}_{u_{y} u_{y} 12}^{G}=\left\langle{ }_{0} \sigma_{x y}^{p, k} \mathbf{Z}_{u_{y} u_{y}}^{p, k}\right\rangle \\
\mathbf{Z}_{u_{z} u_{z} 11}^{G}=\left\langle{ }_{0} \sigma_{x x}^{p, k} \mathbf{Z}_{u_{z} u_{z}}^{p, k}\right\rangle & \mathbf{Z}_{u_{z} u_{z} 22}^{G}=\left\langle{ }_{0} \sigma_{y y}^{p, k} \mathbf{Z}_{u_{z} u_{z}}^{p, k}\right\rangle & \mathbf{Z}_{u_{z} u_{z} 12}^{G}=\left\langle{ }_{0} \sigma_{x y}^{p, k} \mathbf{Z}_{u_{z} u_{z}}^{p, k}\right\rangle
\end{array}
$$

\section{A.2. Stiffness matrix}

$$
\begin{aligned}
\mathbf{K}_{u_{x} u_{x}}= & \mathcal{I}_{u_{x} u_{x}}^{1010} \otimes \mathbf{Z}_{u_{x} u_{x} 11}+\left(\mathcal{I}_{u_{x} u_{x}}^{1001}+\mathcal{I}_{u_{x} u_{x}}^{0110}\right) \otimes \mathbf{Z}_{u_{x} u_{x} 16}+\mathcal{I}_{u_{x} u_{x}}^{0101} \otimes \mathbf{Z}_{u_{x} u_{x} 66} \\
& +\mathcal{I}_{u_{x} u_{u}}^{0000} \otimes \mathbf{Z}_{\partial u_{x} \partial u_{x} 55} \\
\mathbf{K}_{u_{x} u_{y}}= & \mathcal{I}_{u_{x} u_{y}}^{1001} \otimes \mathbf{Z}_{u_{x} u_{y} 12}+\mathcal{I}_{u_{x} u_{y}}^{1010} \otimes \mathbf{Z}_{u_{x} u_{y} 16}+\mathcal{I}_{u_{x} u_{y}}^{0101} \otimes \mathbf{Z}_{u_{x} u_{y} 26} \\
& +\mathcal{I}_{u_{x} u_{y}}^{0110} \otimes \mathbf{Z}_{u_{x} u_{y} 66}+\mathcal{I}_{u_{x} u_{y}}^{0000} \otimes \mathbf{Z}_{\partial u_{x} \partial u_{y} 45} \\
\mathbf{K}_{u_{x} u_{z}}= & \mathcal{I}_{u_{x} u_{z}}^{0010} \otimes \mathbf{Z}_{\partial u_{x} u_{z} 55}+\mathcal{I}_{u_{x} u_{z}}^{0001} \otimes \mathbf{Z}_{\partial u_{x} u_{z} 45}+\mathcal{I}_{u_{x} u_{z}}^{1000} \otimes \mathbf{Z}_{u_{x} \partial u_{z} 13} \\
& +\mathcal{I}_{u_{x} u_{z}}^{0100} \otimes \mathbf{Z}_{u_{x} \partial u_{z} 36} \\
\mathbf{K}_{u_{y} u_{y}}= & \mathcal{I}_{u_{y} u_{y}}^{0101} \otimes \mathbf{Z}_{u_{y} u_{y} 22}+\left(\mathcal{I}_{u_{y} u_{y}}^{0110}+\mathcal{I}_{u_{y} u_{y}}^{1001}\right) \otimes \mathbf{Z}_{u_{y} u_{y} 26}+\mathcal{I}_{u_{y} u_{y}}^{1010} \otimes \mathbf{Z}_{u_{y} u_{y} 66} \\
& +\mathcal{I}_{u_{y} u_{y}}^{0000} \otimes \mathbf{Z}_{\partial u_{y} \partial u_{y} 44} \\
\mathbf{K}_{u_{y} u_{z}}= & \mathcal{I}_{u_{y} u_{z}}^{0010} \otimes \mathbf{Z}_{\partial u_{y} u_{z} 45}+\mathcal{I}_{u_{y} u_{z}}^{0001} \otimes \mathbf{Z}_{\partial u_{y} u_{z} 44}+\mathcal{I}_{u_{y} u_{z}}^{0100} \otimes \mathbf{Z}_{u_{y} \partial u_{z} 23} \\
& +\mathcal{I}_{u_{y} u_{z}}^{1000} \otimes \mathbf{Z}_{u_{y} \partial u_{z} 36} \\
\mathbf{K}_{u_{z} u_{z}}= & \mathcal{I}_{u_{z} u_{z}}^{1010} \otimes \mathbf{Z}_{u_{z} u_{z} 55}+\left(\mathcal{I}_{u_{z} u_{z}}^{10011}+\mathcal{I}_{u_{z} u_{z}}^{0110}\right) \otimes \mathbf{Z}_{u_{z} u_{z} 45}+\mathcal{I}_{u_{z} u_{z}}^{0101} \otimes \mathbf{Z}_{u_{z} u_{z} 44} \\
& +\mathcal{I}_{u_{z} u_{z}}^{0000} \otimes \mathbf{Z}_{\partial u_{z} \partial u_{z} 33} \\
&
\end{aligned}
$$


where the generic thickness integral is

$\mathbf{Z}_{(\partial) u_{r}(\partial) u_{S} R S}=\left\langle\widetilde{C}_{R S}^{p, k} \mathbf{Z}_{u_{z} u_{z}}^{p, k}\right\rangle$

\section{References}

[1] Thornton EA. Thermal structures for aerospace applications. Reston, Virginia: AIAA; 1996.

[2] Hyer MW. Stress analysis of fiber-reinforced composite materials. New York: McGraw-Hill; 1998.

[3] Jones RM. Buckling of bars, plates, and shells. Blacksburg, VA: Bull Ridge Corporation; 2006.

[4] Jones RM. Thermal buckling of uniformly heated unidirectional and symmetric cross-ply laminated fiber-reinforced composite uniaxial in-plane restrained simply supported rectangular plates. Compos Part A: Appl Sci Manuf 2005;36 (10):1355-67.

[5] Nemeth MP. Buckling behavior of long anisotropic plates subjected to fully restrained thermal expansion. TP 2003-212131, NASA; 2003.

[6] Li J, Narita Y, Wang Z. The effects of non-uniform temperature distribution and locally distributed anisotropic properties on thermal buckling of laminated panels. Compos Struct 2015;119:610-9.

[7] Li J, Tian X, Han Z, Narita Y. Stochastic thermal buckling analysis of laminated plates using perturbation technique. Compos Struct 2016;139:1-12.

[8] Duran AV, Fasanella NA, Sundararaghavan, Waas AM. Thermal buckling of composite plates with spatial varying fiber orientations. Compos Struct 2015;124:228-35.

[9] Tauchert TR. Thermal buckling of thick antisymmetric angle-ply laminates. J Therm Stresses 1987;10(2):113-24.

[10] Noor AK, Peters JM. Thermomechanical buckling of multilayered composite plates. J Eng Mech 1992;118(2):351-66.

[11] Kandasamy R, Dimitri R, Tornabene F. Numerical study on the free vibration and thermal buckling behavior of moderately thick functionally graded structures in thermal environments. Compos Struct 2016;157:207-22.

[12] Chang JS, Shyue SY. Thermal buckling analysis of antisymmetric angle-ply laminates based on a higher-order displacement field. Compos Sci Technol 1991;41(2):109-28.

[13] Chang JS. A further study on thermal buckling of simply supported antisymmetric angle-ply laminates in a uniform-temperature field. Compos Sci Technol 1992;43(4):309-15.

[14] Rohwer K. Letter to the editor. Compos Sci Technol 1992;45:181-2.

[15] Dafedar JB, Desai YM. Thermomechanical buckling of laminated composite plates using mixed, higher-order analytical formulation. J Appl Mech 2002;69 (6):790-9.

[16] Lo SH, Zhen W, Cheung YK, Wanji C. Hygrothermal effects on multilayered composite plates using a refined higher order theory. Compos Struct 2010;92 (3):633-46.

[17] Lee J. Thermally induced buckling of laminated composites by a layerwise theory. Comput Struct 1997;65(6):917-22.

[18] Cetkovic M. Thermal buckling of laminated composite plates using layerwise displacement model. Compos Struct 2016;142:238-53.

[19] Ko WL, Jackson RH. Compressive and shear buckling analysis of metal matrix composite sandwich panels under different thermal environments. Compos Struct 1993;25(1-4):227-39.

[20] Libove C, Batdorf SB. A general small-deflection theory for flat sandwich plates. TN 1526, NACA; 1948 .

[21] Babu CS, Kant T. Refined higher order finite element models for thermal buckling of laminated composite and sandwich plates. J Therm Stresses 2000;23(2):111-30.

[22] Kant T, Babu CS. Thermal buckling analysis of skew fibre-reinforced composite and sandwich plates using shear deformable finite element models. Compos Struct 2000;49(1):77-85.

[23] Frostig Y, Thomsen OT. Thermal buckling and postbuckling of sandwich panels with a transversely flexible core. AIAA J 2008;46(8):1976-89.

[24] Pradeep V, Ganesan N, Bhaskar K. Vibration and thermal buckling of composite sandwich beams with viscoelastic core. Compos Struct 2007;81(1):60-9.

[25] Matsunaga $H$. Thermal buckling of cross-ply laminated composite and sandwich plates according to a global higher-order deformation theory. Compos Struct 2005;68(4):439-54.
[26] Matsunaga H. Thermal buckling of angle-ply laminated composite and sandwich plates according to a global higher-order deformation theory. Compos Struct 2006;72(2):177-92.

[27] Matsunaga H. Free vibration and stability of angle-ply laminated composite and sandwich plates under thermal loading. Compos Struct 2007;77 (2):249-62.

[28] Noor AK, Burton WS. Three-dimensional solutions for thermal buckling of multilayered anisotropic plates. J Eng Mech 1992;118(4):683-701.

[29] Noor AK, Burton WS. Three-dimensional solutions for the thermal buckling and sensitivity derivatives of temperature-sensitive muitilayered angle-ply plates. J Appl Mech 1992;59(4):848-56.

[30] Noor AK, Peters AK, Jeanne M, Burton WS. Three-dimensional solutions for initially stressed structural sandwiches. J Eng Mech 1994;120(120):284-303.

[31] Carrera E. A class of two-dimensional theories for anisotropic multilayered plates analysis. Atti Accademia delle Scienze di Torino. Memorie Scienze Fisiche 1995;19:1-39.

[32] Carrera E. Theories and finite elements for multilayered plates and shells: a unified compact formulation with numerical assessment and benchmarking. Arch Comput Methods Eng 2003;10(3):215-96.

[33] Carrera E, Brischetto S. A survey with numerical assessment of classical and refined theories for the analysis of sandwich plates. Appl Mech Rev 2009;62 (1):010803.

[34] Neves AMA, Ferreira AJM, Carrera E, Cinefra M, Roque CMC, Jorge RMN, Mota Soares CM. Static, free vibration and buckling analysis of isotropic and sandwich functionally graded plates using a quasi-3d higher-order shear deformation theory and a meshless technique. Compos Part B: Eng 2013;44 (1):657-74.

[35] Ferreira AJM, Araujo AL, Neves AMA, Rodrigues JD, Carrera E, Cinefra M, Mota Soares CM. A finite element model using a unified formulation for the analysis of viscoelastic sandwich laminate. Compos Part B: Eng 2013;45(1):1258-64.

[36] D'Ottavio M. A Sublaminate Generalized Unified Formulation for the analysis of composite structures. Compos Struct 2016;142:187-99.

[37] D'Ottavio M, Dozio L, Vescovini R, Polit O. Bending analysis of composite laminated and sandwich structures using sublaminate variable-kinematic Ritz models. Compos Struct 2016;155:45-62.

[38] Nali P, Carrera E. Accurate buckling analysis of composite layered plates with combined thermal and mechanical loadings. J Therm Stresses 2013:36:1-18.

[39] Fazzolari FA, Carrera E. Thermo-mechanical buckling analysis of anisotropic multilayered composite and sandwich plates by using refined variablekinematics theories. J Therm Stresses 2013;36(4):321-50.

[40] Fazzolari A. Natural frequencies and critical temperatures of functionally graded sandwich plates subjected to uniform and non-uniform temperature distributions. Compos Struct 2015;121:197-210.

[41] Carrera E. Temperature profile influence on layered plates response considering classical and advanced theories. AIAA J 2002;40(9):1885-96.

[42] Carrera E. Transverse normal strain effect on thermal stress analysis of homogeneous and layered plates. AIAA J 2005;43(10):2232-42.

[43] Demasi L. $\infty^{6}$ mixed plate theories based on the Generalized Unified Formulation. Part I: Governing equations. Compos Struct 2009;87(1):1.

[44] Demasi L. $\infty^{6}$ mixed plate theories based on the Generalized Unified Formulation. Part II: Layerwise theories. Compos Struct 2009;87(1):12-22.

[45] Demasi L. $\infty^{6}$ mixed plate theories based on the Generalized Unified Formulation. Part III: advanced mixed high order shear deformation theories. Compos Struct 2009;87(3):83-194.

[46] Dozio L, Carrera E. Ritz analysis of vibrating rectangular and skew multilayered plates based on advanced variable-kinematic models. Compos Struct 2012;94 (6):2118-28.

[47] Dozio L. Refined 2-D theories for free vibration analysis of annular plates: Unified Ritz formulation and numerical assessment. Comput Struct 2015;147:250-8.

[48] Vescovini R, Dozio L. A variable-kinematic model for variable stiffness plates: vibration and buckling analysis. Compos Struct 2016;142:15-26.

[49] Reddy JN. Mechanics of laminated composite plates and shells: theory and analysis. Boca Raton: CRC Press; 2004

[50] Carrera E, Brischetto S. Analysis of thickness locking in classical, refined and mixed multilayered plate theories. Compos Struct 2008;82(4):549-62. 